\title{
Regularity of Homogenized Boundary Data in Periodic Homogenization of Elliptic Systems
}

\author{
Zhongwei Shen* Jinping Zhuge ${ }^{\dagger}$
}

\begin{abstract}
This paper is concerned with periodic homogenization of second-order elliptic systems in divergence form with oscillating Dirichlet data or Neumann data of first order. We prove that the homogenized boundary data belong to $W^{1, p}$ for any $1<p<\infty$. In particular, this implies that the boundary layer tails are Hölder continuous of order $\alpha$ for any $\alpha \in(0,1)$.
\end{abstract}

MSC2010: 35B27, 74Q05.

Keywords: Homogenization; Boundary Layers; Oscillating Boundary Data.

\section{Introduction}

In this paper we consider the uniformly elliptic operator with periodically oscillating coefficients

$$
\mathcal{L}_{\varepsilon}=-\operatorname{div}(A(x / \varepsilon) \nabla)=-\frac{\partial}{\partial x_{i}}\left\{a_{i j}^{\alpha \beta}\left(\frac{x}{\varepsilon}\right) \frac{\partial}{\partial x_{j}}\right\},
$$

where $1 \leq i, j \leq d, 1 \leq \alpha, \beta \leq m$, and $0<\varepsilon \leq 1$. Throughout we assume that the coefficient matrix $A$ satisfies the following conditions:

- Ellipticity: there exists $\lambda>0$ such that

$$
\lambda|\xi|^{2} \leq a_{i j}^{\alpha \beta} \xi_{i}^{\alpha} \xi_{j}^{\beta} \leq \lambda^{-1}|\xi|^{2} \quad \text { for any } \xi=\left(\xi_{i}^{\alpha}\right) \in \mathbb{R}^{m \times d} ;
$$

- Periodicity: $A$ is 1 -periodic, that is

$$
A(y+z)=A(y) \quad \text { for any } y \in \mathbb{R}^{d} \text { and } z \in \mathbb{Z}^{d} ;
$$

- Smoothness:

$$
a_{i j}^{\alpha \beta} \in C^{\infty}\left(\mathbb{T}^{d}\right) \quad \text { for } 1 \leq \alpha, \beta \leq m \text { and } 1 \leq i, j \leq d .
$$

*Supported in part by NSF grant DMS-1600520.

${ }^{\dagger}$ Supported in part by NSF grant DMS-1600520. 
Recently, there has been considerable interest in the study of homogenization of Dirichlet problem with oscillating boundary data,

$$
\left\{\begin{aligned}
\mathcal{L}_{\varepsilon}\left(u_{\varepsilon}\right) & =0 & & \text { in } \Omega, \\
u_{\varepsilon}(x) & =f(x, x / \varepsilon) & & \text { on } \partial \Omega,
\end{aligned}\right.
$$

where $f(x, y)$ is 1-periodic in $y$ [14, 15, 20, 17, 3, 4, 5, 2, 8, 21, 23] (also see earlier work in [18, 19, 6] as well as related work for nonlinear elliptic equations in [11, 10, 13, 12]). In particular, under the assumption that $\Omega$ is a smooth and strictly convex domain in $\mathbb{R}^{d}$, it was proved in [15] that the homogenized problem for (1.4) is given by

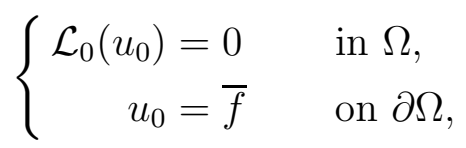

where $\mathcal{L}_{0}$ is the usual homogenized operator and $\bar{f}$ is a function whose value at $x \in \partial \Omega$ depends only on $A, f(x, \cdot)$ and the outward normal $n$ to $\partial \Omega$ at $x$. Moreover, a convergence rate for $\left\|u_{\varepsilon}-u_{0}\right\|_{L^{2}(\Omega)}$ was established in [15]. The near sharp convergence rates were obtained in [8] for $d \geq 4$ and in [21] for $d=2$ or 3. Furthermore, the present authors in [21] considered the Neumann problem with first-order oscillating boundary data,

$$
\left\{\begin{aligned}
\mathcal{L}_{\varepsilon}\left(u_{\varepsilon}\right) & =0 & & \text { in } \Omega, \\
\frac{\partial u_{\varepsilon}}{\partial \nu_{\varepsilon}} & =T_{i j} \cdot \nabla_{x}\left\{g_{i j}(x, x / \varepsilon)\right\} & & \text { on } \partial \Omega,
\end{aligned}\right.
$$

where $T_{i j}=n_{i} e_{j}-n_{j} e_{j}$ is a tangential vector field on $\partial \Omega$ and $\left\{g_{i j}(x, y)\right\}$ are 1-periodic in $y$. It was proved in [21] that if $\Omega$ is smooth and strictly convex, the homogenized problem for (1.6) is given by

$$
\left\{\begin{aligned}
\mathcal{L}_{0}\left(u_{0}\right) & =0 & & \text { in } \Omega, \\
\frac{\partial u_{0}}{\partial \nu_{0}} & =T_{i j} \cdot \nabla_{x} \bar{g}_{i j} & & \text { on } \partial \Omega,
\end{aligned}\right.
$$

where $\frac{\partial u_{0}}{\partial \nu_{0}}$ denotes the conormal derivative of $u_{0}$ associated with $\mathcal{L}_{0}$, and $\left\{\bar{g}_{i j}\right\}$ are functions on $\partial \Omega$ whose value at $x \in \partial \Omega$ depend only on $A,\left\{g_{i j}(x, \cdot)\right\}$ and $n(x)$. Assume that $\int_{\Omega} u_{\varepsilon}=\int_{\Omega} u_{0}=0$. The near optimal rate of convergence for $\left\|u_{\varepsilon}-u_{0}\right\|_{L^{2}(\Omega)}$ was also established in [21] for $d \geq 3$. In [23] the second author investigated the case of nonconvex domains and extended the results in [8, 21] for Dirichlet problems to certain domains of finite type. We point out that one of main motivations for studying boundary value problems (1.4) and (1.6) with oscillating data is its applications to the higher-order convergence in the two-scale expansions of solutions to boundary value problems with non-oscillating boundary data.

Our primary concern in this paper is the regularity of the homogenized data $\bar{f}$ in (1.5) and $\left\{\bar{g}_{i j}\right\}$ in (1.7). Under the assumption that $\Omega$ is smooth and strictly convex, it was proved in [15] that $\nabla_{\tan } \bar{f} \in L^{p, \infty}(\partial \Omega)$ with $p=\frac{d-1}{2}$. The result was improved in [8] to $\nabla_{\tan } \bar{f} \in L^{p, \infty}(\partial \Omega)$ with $p=\frac{2(d-1)}{3}$ if $d \geq 3$, and to $\bar{f} \in W^{1, p}(\partial \Omega)$ for any $p<\frac{2}{3}$ if 
$d=2$. Further improvement was made in [21], where we proved that $\bar{f} \in W^{1, p}(\partial \Omega)$ for any $p<d-1$ and $d \geq 2$. In [21] we also obtained the regularity estimate $\bar{g}_{i j} \in W^{1, p}(\partial \Omega)$ for the Neumann problem (1.6), where $p<d-1$ and $d \geq 3$.

The following two theorems are the main results of this paper.

Theorem 1.1 (Dirichlet Data). Assume that A satisfies (1.1), (1.2) and (1.3). Let $\Omega$ be a smooth and strictly convex domain in $\mathbb{R}^{d}$. Let $\bar{f}$ denote the homogenized data in (1.5). Then

$$
\|\bar{f}\|_{W^{1, p}(\partial \Omega)} \leq C_{p}\left(\int_{\mathbb{T}^{d}}\|f(\cdot, y)\|_{C^{1}(\partial \Omega)}^{2} d y\right)^{1 / 2} \quad \text { for any } 1<p<\infty,
$$

where $C_{p}$ depends only on $d, m, \lambda, p$, and $\|A\|_{C^{k}\left(\mathbb{T}^{d}\right)}$ for some $k=k(d, p)>1$.

Theorem 1.2 (Neumann Data). Assume that A satisfies (1.1), (1.2) and (1.3). Let $\Omega$ be a smooth and strictly convex domain in $\mathbb{R}^{d}$. Let $\bar{g}=\left(\bar{g}_{i j}\right)$ denote the homogenized data in (1.7). Then

$$
\|\bar{g}\|_{W^{1, p}(\partial \Omega)} \leq C_{p}\left(\int_{\mathbb{T}^{d}}\|g(\cdot, y)\|_{C^{1}(\partial \Omega)}^{2} d y\right)^{1 / 2} \quad \text { for any } 1<p<\infty,
$$

where $C_{p}$ depends only on $d, m, \lambda, p$, and $\|A\|_{C^{k}\left(\mathbb{T}^{d}\right)}$ for some $k=k(d, p)>1$.

It follows from regularity estimates (1.8) and (1.9) that the homogenized data $\bar{f}$ and $\bar{g}=\left(\bar{g}_{i j}\right)$ are Hölder continuous of order $\alpha$ for any $\alpha \in(0,1)$. We should point out that the assumption that $\Omega$ is strictly convex is not essential for Theorems 1.1 and 1.2. In fact, the proof goes through as long as one has $[\varkappa(n(x))]^{-1} \in L^{q}(\partial \Omega)$ for some $q>0$ (see (1.11) for the definition of $\varkappa$ ). Consequently, the conclusions of Theorems 1.1 and 1.2 continue to hold for the domains of finite type considered in [23].

We mention several related work regarding the continuity of homogenized data. In [1, under the additional assumption that $A$ is independent of some rational direction $\nu_{0}$, it was proved that the homogenized Dirichlet data has a unique continuous extension to the set $\left\{x \in \partial \Omega: n(x) \cdot \nu_{0} \neq 0\right\}$. The problem of Hölder continuity was also studied in [10, 12] for second-order nonlinear elliptic equations of form $F\left(D^{2} u_{\varepsilon}, x / \varepsilon\right)=0$. In particular, it was shown in [12] that if the homogenized operator $\bar{F}$ is either rotational invariant or linear, then the homogenized Dirichlet data is Hölder continuous, and that the homogenized data may be discontinuous in general. Note that the linear elliptic equations in non-divergence form may be written in a divergence form with $\operatorname{div}(A)=0$. In this case, the first-order correctors are trivial and as a result, it is easy to see that the homogenized data is smooth if $\Omega$ is smooth and satisfies some geometric conditions. As far as we know, the continuity of the homogenized data in the general case of elliptic equations in divergence form is not known previously.

We now describe our general approach to Theorems 1.1 and 1.2 as well as some of the key estimates in the proof. Our starting point for the proof of Theorem 1.1 is a formula for the homogenized data $\bar{f}$ discovered in [8]. See Theorem 2.5 , This formula reduces the 
problem to the study of the dependance on $n \in \mathbb{S}^{d-1}$ of solutions $V_{n}=V_{n}(\theta, t)$ to the Dirichlet problem,

$$
\left\{\begin{array}{rr}
-\left(\begin{array}{c}
N_{n}^{T} \nabla_{\theta} \\
\partial_{t}
\end{array}\right) \cdot B_{n}\left(\begin{array}{c}
N_{n}^{T} \nabla_{\theta} \\
\partial_{t}
\end{array}\right) V_{n}=0 & \text { in } \mathbb{T}^{d} \times \mathbb{R}_{+}, \\
V_{n}(\theta, 0)=\phi(\theta) & \text { on } \mathbb{T}^{d} \times\{0\}
\end{array}\right.
$$

where $\phi \in C^{\infty}\left(\mathbb{T}^{d} ; \mathbb{R}^{m}\right), B_{n}=B_{n}(\theta, t)=M_{n}^{T} A^{*}(\theta-t n) M_{n}$, and $M_{n}$ is a $d \times d$ orthogonal matrix whose first $d-1$ columns are given by $N_{n}$ and whose last column is $-n$.

To describe our key estimates, we need to introduce some notations. A unit vector $n=\left(n_{1}, n_{2}, \cdots, n_{d}\right) \in \mathbb{S}^{d-1}$ is called rational if $n \in \mathbb{R} \mathbb{Z}^{d}$ and called irrational otherwise. Moreover, a unit vector $n$ is called Diophantine if there exists some constant $C>0$ such that

$$
|(I-n \otimes n) \xi| \geq C|\xi|^{-2} \quad \text { for all } \xi \in \mathbb{Z}^{d} \backslash\{0\} .
$$

Denote by $\varkappa=\varkappa(n)$ the Diophantine constant, which is defined as the largest constant validating (1.11). We use $\mathbb{S}_{R}^{d-1}, \mathbb{S}_{I}^{d-1}$ and $\mathbb{S}_{D}^{d-1}$ to represent the sets of rational, irrational and Diophantine unit vectors, respectively. Note that $\mathbb{S}_{D}^{d-1}$ is a subset of $\mathbb{S}_{I}^{d-1}$ and has full surface measure of $\mathbb{S}^{d-1}$.

Let $n, \widetilde{n} \in \mathbb{S}_{D}^{d-1}$. We will show in Section 2 that for any $\sigma \in(0,1)$,

$$
\left(\int_{\mathbb{T}^{d}}\left|\partial_{t} V_{n}(\theta, 0)-\partial_{t} V_{\widetilde{n}}(\theta, 0)\right|^{2} d \theta\right)^{1 / 2} \leq C_{\sigma} \varkappa^{-\sigma}|n-\widetilde{n}|,
$$

where $\varkappa=\max \{\varkappa(n), \varkappa(\widetilde{n})\}$ and $C_{\sigma}$ depends only on $d, m, \sigma, \lambda,\|A\|_{C^{k}\left(\mathbb{T}^{d}\right)}$ and $\|\phi\|_{C^{k}\left(\mathbb{T}^{d}\right)}$ for some $k=k(d, \sigma)>1$. Theorem 1.1 follows from (1.12) by using the representation formula mentioned above and an approximation argument.

To prove (1.12), besides the energy estimates established in [14, 15, 8], one needs to fully take advantage of the fact that if

$$
u^{s}(x)=V_{n}(x-(x \cdot n) n-s n,-x \cdot n-s),
$$

then $u^{s}$ is a solution of the Dirichlet problem in a half-space,

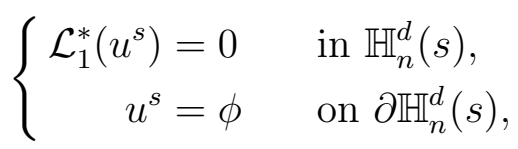

where $\mathbb{H}_{n}^{d}(s)=\mathbb{H}_{n}^{d}-s n$ and $\mathbb{H}_{n}^{d}=\left\{x \in \mathbb{R}^{d}: x \cdot n<0\right\}$ is the half-space whose boundary contains the origin and with outward normal $n$. This allows us to apply the large-scale boundary regularity estimates for the operator $\mathcal{L}_{1}^{*}$. The technique was already used in [15, 8] to establish the boundedness of $V_{n}$ and in [21] for a crucial weighted norm inequality. Here, among other things, we apply the technique to establish the boundedness of $\nabla_{\theta} V_{n}$ as well as some pointwise decay estimates for $\partial_{t} V_{n}$ and $N_{n}^{T} \nabla_{\theta} V_{n}$. 
We remark that the asymptotic behavior of the solution $u^{s}$ of (1.14) as $x \cdot n \rightarrow-\infty$ is well understood thanks to [18, 6, 14, 15, 20, 2]. In particular, if $n$ is irrational, it was shown in [20] that there exists a constant vector $\mu^{*}(n, \phi) \in \mathbb{R}^{m}$ independent of $s$ such that

$$
\mu^{*}(n, \phi)=\lim _{x \cdot n \rightarrow-\infty} u^{s}(x)
$$

though the rate of convergence could be arbitrarily slow in general. On the other hand, if $n$ is rational [18, 6], the above limit depends on $s$ and possesses an exponential rate of convergence. The mapping $\mu: \mathbb{S}_{I}^{d-1} \times C^{\infty}\left(\mathbb{T}^{d} ; \mathbb{R}^{m}\right) \mapsto \mathbb{R}^{m}$ defined via (1.15), but with $\mathcal{L}_{1}^{*}$ replaced by $\mathcal{L}_{1}$, is called the boundary layer tail (BLT) for Dirichlet problems associated with $\mathcal{L}_{1}$. It follows from [15] that

$$
\bar{f}(x)=\mu(n(x), f(x, \cdot)), \quad \text { if } n(x) \in \mathbb{S}_{D}^{d-1} .
$$

Thus, by Theorem 1.1 $\|\mu(\cdot, \phi)\|_{W^{1, p\left(\mathbb{S}^{d-1}\right)}} \leq C\|\phi\|_{L^{2}\left(\mathbb{T}^{d}\right)}$ for any $1<p<\infty$. Consequently, for any $0<\alpha<1, \mu(\cdot, \phi)$ extends to a Hölder continuous function of order $\alpha$ on $\mathbb{S}^{d-1}$ and

$$
|\mu(n, \phi)-\mu(\widetilde{n}, \phi)| \leq C_{\alpha}|n-\widetilde{n}|^{\alpha}\|\phi\|_{L^{2}\left(\mathbb{T}^{d}\right)} \quad \text { for any } n, \widetilde{n} \in \mathbb{S}^{d-1},
$$

where $C_{\alpha}$ depends only on $d, m, \alpha$ and $A$.

Our approach to Theorem 1.2 for Neumann problems is similar to that used for Theorem 1.1. The starting point is a formula for the homogenized data $\left\{\bar{g}_{i j}\right\}$ obtained in [21]. See Theorem 3.3. As in the case of Dirichlet problems, this formula reduces the problem to the study of the dependence in $n \in \mathbb{S}^{d-1}$ of solutions $U_{n}=U_{n}(\theta, t)$ to the Neumann problem,

$$
\left\{\begin{array}{rr}
-\left(\begin{array}{c}
N_{n}^{T} \nabla_{\theta} \\
\partial_{t}
\end{array}\right) \cdot B_{n}\left(\begin{array}{c}
N_{n}^{T} \nabla_{\theta} \\
\partial_{t}
\end{array}\right) U_{n}=0 & \text { in } \mathbb{T}^{d} \times \mathbb{R}_{+}, \\
-e_{d+1} \cdot B_{n}\left(\begin{array}{c}
N_{n}^{T} \nabla_{\theta} \\
\partial_{t}
\end{array}\right) U_{n}=T_{n} \cdot \nabla_{\theta} \phi & \text { on } \mathbb{T}^{d} \times\{0\},
\end{array}\right.
$$

where $T_{n} \in \mathbb{R}^{d},\left|T_{n}\right| \leq 1$ and $T_{n} \cdot n=0$. Let $n, \widetilde{n} \in \mathbb{S}_{D}^{d-1}$. We will show in Section 3 that for any $\sigma \in(0,1)$,

$$
\left(\int_{\mathbb{T}^{d}}\left|\nabla_{\theta} U_{n}(\theta, 0)-\nabla_{\theta} U_{\widetilde{n}}(\theta, 0)\right|^{2} d \theta\right)^{1 / 2} \leq C_{\sigma} \varkappa^{-\sigma}|n-\widetilde{n}|,
$$

where $\varkappa=\max \{\varkappa(n), \varkappa(\widetilde{n})\}$ and $C_{\sigma}$ depends only on $d, m, \sigma, \lambda,\|A\|_{C^{k}\left(\mathbb{T}^{d}\right)}$ and $\|\phi\|_{C^{k}\left(\mathbb{T}^{d}\right)}$ for some $k=k(d, \sigma)>1$. Theorem 1.2 follows from (1.19) and the representation formula mentioned above. Finally, we point out that the key estimates in the proof of (1.19) rely on the observation that if $u^{s}(x)=U_{n}(x-(x \cdot n) n-s n,-x \cdot n-s)$, then $u^{s}$ is a solution to the Neumann problem,

$$
\mathcal{L}_{1}^{*}\left(u^{s}\right)=0 \quad \text { in } \mathbb{H}_{n}^{d}(s) \quad \text { and } \quad \frac{\partial u^{s}}{\partial \nu_{1}^{*}}=T_{n} \cdot \nabla_{x} \phi \quad \text { on } \partial \mathbb{H}_{n}^{d}(s)
$$

We refer the reader to Section 3 for details. 


\section{Regularity for Dirichlet problems}

Assume that $A$ satisfies conditions (1.1)-(1.3). For $1 \leq j \leq d$ and $1 \leq \beta \leq m$, let $\chi=\left(\chi_{j}^{\beta}\right)=\left(\chi_{j}^{1 \beta}, \chi_{j}^{2 \beta}, \cdots, \chi_{j}^{m \beta}\right)$ denote the correctors for $\overline{\mathcal{L}}_{\varepsilon}$. By definition they are 1-periodic functions satisfying the equation $\mathcal{L}_{1}\left(\chi_{j}^{\beta}+P_{j}^{\beta}\right)=0$ in $\mathbb{R}^{d}$ with $\int_{\mathbb{T}^{d}} \chi_{j}^{\beta}=0$, where $P_{j}^{\beta}(x)=x_{j} e^{\beta}$. The homogenized operator is given by $\mathcal{L}_{0}=-\operatorname{div}(\widehat{A} \nabla)$, where the homogenized matrix $\widehat{A}=\left(\widehat{a}_{i j}^{\alpha \beta}\right)$ is defined by

$$
\widehat{a}_{i j}^{\alpha \beta}=\int_{\mathbb{T}^{d}}\left\{a_{i j}^{\alpha \beta}+a_{i k}^{\alpha \gamma} \frac{\partial}{\partial y_{k}}\left(\chi_{j}^{\gamma \beta}\right)\right\} .
$$

We also introduce the adjoint operator $\mathcal{L}_{\varepsilon}^{*}=-\operatorname{div}\left(A^{*}(x / \varepsilon) \nabla\right)$, where $A^{*}=\left(a_{i j}^{* \alpha \beta}\right)$ with $a_{i j}^{* \alpha \beta}=a_{j i}^{\beta \alpha}$. Note that $A^{*}$ also satisfies (1.1)-(1.3). Let $\chi^{*}=\left(\chi_{j}^{* \beta}\right)$ denote the correctors for $\mathcal{L}_{\varepsilon}^{*}$.

The solvability of the Dirichlet problem (1.14) is not obvious, since $\mathbb{H}_{n}^{d}(s)$ is unbounded. Nevertheless, by using Lipschitz estimates in [9] and an approximation argument, one may establish the existence of the Poisson kernel in a half-space and hence the solvability of (1.14) via the Poisson integral formula.

Theorem 2.1. Let $\Omega=\mathbb{H}_{n}^{d}(s)$ for some $n \in \mathbb{S}^{d-1}$ and $s \in \mathbb{R}$. Then, for any bounded continuous function $\phi$ in $\mathbb{R}^{d}$, there exists a unique bounded function $u$ in $C^{\infty}\left(\Omega ; \mathbb{R}^{m}\right) \cap$ $C\left(\bar{\Omega} ; \mathbb{R}^{m}\right)$ such that

$$
\mathcal{L}_{1}^{*}(u)=0 \quad \text { in } \Omega \quad \text { and } \quad u=\phi \quad \text { on } \partial \Omega .
$$

Moreover, the solution may be represented by

$$
u(x)=\int_{\partial \Omega} P^{*}(x, y) \phi(y) d \sigma(y),
$$

where the Poisson kernel $P^{*}=P^{*}(x, y)$ satisfies

$$
\begin{gathered}
\left|P^{*}(x, y)\right| \leq \frac{C \delta(x)}{|x-y|^{d}}, \\
\left|\nabla_{x} P^{*}(x, y)\right| \leq \frac{C \min \{|x-y|, \delta(x)\}}{|x-y|^{d+1}}
\end{gathered}
$$

for any $x \in \Omega$ and $y \in \partial \Omega, \delta(x)=\operatorname{dist}\left(x, \partial \mathbb{H}_{n}^{d}(s)\right)=|s+x \cdot n|$, and $C$ depends only on $d, m, \lambda$, and some Hölder norm of $A$ on $\mathbb{T}^{d}$.

Proof. The theorem was proved in [15, Proposition 2.5].

Remark 2.2. By the boundary Lipschitz estimates [9] and the Cacciopoli inequality, the uniqueness holds under the sublinear growth condition: $|u(x)| \leq C_{0}(1+\delta(x))^{\alpha}$ for some $C_{0}>0$ and $\alpha \in(0,1)$. Also, it follows readily from (2.3) that the Miranda-Agmon maximum principle,

$$
\|u\|_{L^{\infty}(\Omega)} \leq C\|\phi\|_{L^{\infty}(\partial \Omega)}
$$

holds, where $C$ depends only on $d, m, \lambda$, and some Hölder norm of $A$ on $\mathbb{T}^{d}$. 
An alternative way to establish the solvability of (1.14) for periodic data $\phi$ is to lift the problem to a $(d+1)$-dimensional problem in the upper half-space. Fix $n \in \mathbb{S}^{d-1}$. Let $M=(N,-n)$ be a $d \times d$ orthogonal matrix such that the last column is $-n$ and the first $d-1$ column is a $d \times(d-1)$ matrix $N$. Now we seek a solution $u$ of (1.14) in a particular form

$$
u^{s}(x)=V(x-(x \cdot n) n-s n,-x \cdot n-s) .
$$

It is not hard to see that $V=V(\theta, t)$ has to satisfy the following lifted degenerate system,

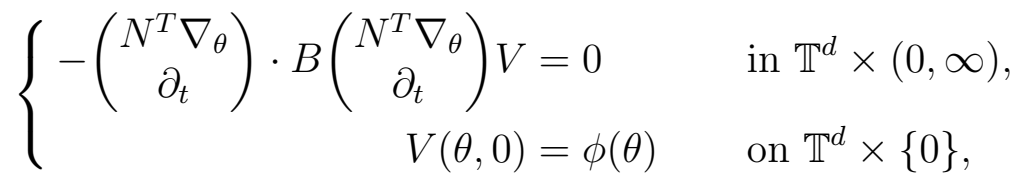

where $B(\theta, t)=M^{T} A^{*}(\theta-t n) M$. Note that $M M^{T}=I$ implies $I=N N^{T}+n \otimes n$. It follows that

$$
M\left(\begin{array}{c}
N^{T} \nabla_{\theta} \\
\partial_{t}
\end{array}\right)=(I-n \otimes n) \nabla_{\theta}-n \partial_{t}
$$

Thus, the solution $V$ is independent of the choice of $N$.

The well-posedness of (2.7) was given by [15, Propositions 2.1 and 2.6].

Lemma 2.3. Let $n \in \mathbb{S}^{d-1}$. Then, for any $\phi \in C^{\infty}\left(\mathbb{T}^{d} ; \mathbb{R}^{m}\right)$, the system (2.7) has a smooth solution $V=V(\theta, t)$ satisfying

$$
\left(\int_{0}^{\infty} \int_{\mathbb{T}^{d}}\left(\left|N^{T} \nabla_{\theta} \partial_{\theta}^{\alpha} \partial_{t}^{j} V\right|^{2}+\left|\partial_{\theta}^{\alpha} \partial_{t}^{1+j} V\right|^{2} d \theta\right) d t\right)^{1 / 2} \leq C\|\phi\|_{C^{|\alpha|+j+1}\left(\mathbb{T}^{d}\right)},
$$

where $|\alpha|, j \geq 0$, and $C$ depends only on $d, m,|\alpha|, j$ and $A$. Moreover, if $n \in \mathbb{S}_{D}^{d-1}$ with Diophantine constant $\varkappa>0$, then there exists a constant $V_{\infty}$ such that for any $|\alpha|, j$, $\ell \geq 0$

$$
\left|N^{T} \nabla_{\theta} \partial_{\theta}^{\alpha} \partial_{t}^{j} V\right|+\left|\partial_{\theta}^{\alpha} \partial_{t}^{1+j} V\right|+\varkappa\left|\partial_{\theta}^{\alpha}\left(V-V_{\infty}\right)\right| \leq \frac{C_{\ell}\|\phi\|_{C^{k}\left(\mathbb{T}^{d}\right)}}{(1+\varkappa t)^{\ell}}
$$

where $k=k(|\alpha|, j, \ell, d)$ and $C_{\ell}$ depends only on $d, m,|\alpha|, j, \ell$ and $A$.

Remark 2.4. The solution of (1.14) given by Theorem 2.1 coincides with the solution of (1.14) given by Lemma 2.3 via (2.6) for any $n \in \mathbb{S}^{d-1}$. To see this, let $w(x)=$ $u^{s}(x)-V(x-(x \cdot n) n-s n,-x \cdot n-s)$. Clearly, $w$ satisfies

$$
\left\{\begin{aligned}
\mathcal{L}_{1}^{*} w=0 & \text { in } \mathbb{H}_{n}^{d}(s) \\
w=0 & \text { on } \partial \mathbb{H}_{n}^{d}(s) .
\end{aligned}\right.
$$


Since $u^{s}$ is bounded and $V$ satisfies

$$
\begin{aligned}
|V(\theta, t)| & =\left|\int_{0}^{t} \partial_{\rho} V(\theta, \rho) d \rho+\phi(\theta)\right| \\
& \leq\|\phi\|_{\infty}+t^{1 / 2}\left(\int_{0}^{\infty}\left|\partial_{\rho} V(\theta, \rho)\right|^{2} d \rho\right)^{1 / 2} \\
& \leq\|\phi\|_{\infty}+C t^{1 / 2}\left(\int_{0}^{\infty}\left\|\partial_{\rho} V(\cdot, \rho)\right\|_{H^{k}\left(\mathbb{T}^{d}\right)}^{2} d \rho\right)^{1 / 2} \\
& \leq\|\phi\|_{\infty}+C t^{1 / 2}\|f\|_{H^{k+2}\left(\mathbb{T}^{d}\right)}
\end{aligned}
$$

for some $k \geq 1$, we conclude that $w$ is of sublinear growth as $|x \cdot n| \rightarrow \infty$. Thus, by Remark 2.2, we obtain $w \equiv 0$.

Now we give an explicit expression for $\bar{f}(x)$ if $n(x) \in \mathbb{S}_{D}^{d-1}$. For $1 \leq k \leq d$ and $1 \leq \beta \leq m$, let $V_{n, k}^{\beta}=V_{n, k}^{\beta}(\theta, t)$ denote the solution of the following Dirichlet problem,

$$
\left\{\begin{array}{rlr}
-\left(\begin{array}{c}
N^{T} \nabla_{\theta} \\
\partial_{t}
\end{array}\right) \cdot B_{n}\left(\begin{array}{c}
N^{T} \nabla_{\theta} \\
\partial_{t}
\end{array}\right) V_{n, k}^{\beta} & =0 & \text { in } \mathbb{T}^{d} \times(0, \infty), \\
V_{n, k}^{\beta} & =-\chi_{k}^{* \beta} & \text { on } \mathbb{T}^{d} \times\{0\},
\end{array}\right.
$$

where $\chi_{k}^{* \beta}$ are the correctors for $\mathcal{L}_{\varepsilon}^{*}, B_{n}=M^{T} A^{*}(\theta-t n) M$, and $M=(N,-n)$ is an orthogonal matrix.

Theorem 2.5. Let $x \in \partial \Omega$. Suppose that $n=n(x) \in \mathbb{S}_{D}^{d-1}$. Let $V_{n}(\theta, t)$ be the solution of (2.12). Then

$$
\bar{f}^{\alpha}(x)=\int_{\mathbb{T}^{d}} h^{\alpha \beta}\left[\delta^{\gamma \beta}+\frac{\partial}{\partial \theta_{\ell}} \chi_{k}^{* \gamma \beta}(\theta) n_{\ell} n_{k}-\partial_{t} V_{n, k}^{\gamma \beta}(\theta, 0) \cdot n_{k}\right] a_{i j}^{\gamma \nu}(\theta) n_{i} n_{j} f^{\nu}(x, \theta) d \theta
$$

for $1 \leq \alpha \leq m$, where $h=\left(h^{\alpha \beta}\right)$ denotes the inverse matrix of the $m \times m$ matrix $\left(\widehat{a}_{i j}^{* \alpha \beta} n_{i} n_{j}\right)$.

Proof. This was proved in [8] (also see [23]).

We now turn to the proof of Theorem 1.1. The key step is to prove the following.

Theorem 2.6. Fix $\sigma \in(0,1)$. Let $x, y \in \partial \Omega$ and $|x-y| \leq c_{0}$. Suppose that $n(x)$, $n(y) \in \mathbb{S}_{D}^{d-1}$. Then

$$
|\bar{f}(x)-\bar{f}(y)| \leq C_{\sigma^{\prime}} \varkappa^{-\sigma}|x-y|\left(\int_{\mathbb{T}^{d}}\|f(\cdot, y)\|_{C^{1}(\partial \Omega)}^{2} d y\right)^{1 / 2},
$$

where $\varkappa=\max \{\varkappa(n(x)), \varkappa(n(y))\}$ and $C_{\sigma}$ depends only on $d, m, \sigma, \lambda$, and $\|A\|_{C^{k}\left(\mathbb{T}^{d}\right)}$ for some $k=k(d, \sigma) \geq 1$.

To prove Theorem 2.6, in view of the formula (2.13), we investigate the continuity in $n$ of the solution to the Dirichlet problem (2.7). 
Lemma 2.7. For $\phi \in C^{\infty}\left(\mathbb{T}^{d} ; \mathbb{R}^{m}\right)$, let $V$ be the solution of (2.7), given by Lemma 2.3, with $n \in \mathbb{S}^{d-1}$. Then

$$
\left|N^{T} \nabla_{\theta} V\right|+\left|\partial_{t} V\right| \leq \frac{C\|\phi\|_{C^{2}\left(\mathbb{T}^{d}\right)}}{1+t}
$$

where $C$ depends only on $d, m$ and $A$. Moreover, for any $|\alpha|, j \geq 0$ and $0<\sigma<1$,

$$
\left|N^{T} \nabla_{\theta} \partial_{\theta}^{\alpha} \partial_{t}^{j} V\right|+\left|\partial_{\theta}^{\alpha} \partial_{t}^{1+j} V\right| \leq \frac{C_{\sigma}\|\phi\|_{C^{k}\left(\mathbb{T}^{d}\right)}}{(1+t)^{1-\sigma}}
$$

where $k=k(|\alpha|, j, \sigma, d)$ and $C_{\sigma}$ depends only on $d, m,|\alpha|, j, \sigma$ and $A$.

Proof. Let $u^{s}$ be given by (2.6). Then

$$
\left\{\begin{aligned}
\mathcal{L}_{1}^{*} u^{s}=0 & \text { in } \mathbb{H}_{n}^{d}(s), \\
u^{s}=\phi & \text { on } \partial \mathbb{H}_{n}^{d}(s) .
\end{aligned}\right.
$$

It follows from (2.6) that

$$
V(\theta, t)=u^{-\theta \cdot n}(\theta-t n) \quad \text { for all }(\theta, t) \in \mathbb{T}^{d} \times \mathbb{R}_{+} .
$$

Thanks to the fact $N^{T} \nabla_{\theta}(\theta \cdot n)=0$, the last equality implies that

$$
\left\{\begin{aligned}
N^{T} \nabla_{\theta} V(\theta, t) & =N^{T} \nabla_{x} u^{-\theta \cdot n}(\theta-t n), \\
\partial_{t} V(\theta, t) & =-n \cdot \nabla_{x} u^{-\theta \cdot n}(\theta-t n) .
\end{aligned}\right.
$$

As a result, estimates for $N^{T} \nabla_{\theta} V$ and $\partial_{t} V$ may be reduced to the corresponding estimates for $u^{s}$.

It follows from the presentation of Poisson integral (2.2) and the pointwise estimate (2.4) that

$$
\left|\nabla u^{s}(x)\right| \leq \frac{C\|\phi\|_{\infty}}{|s+x \cdot n|} .
$$

To deal with the case where $|s+x \cdot n|=\operatorname{dist}\left(x, \partial \mathbb{H}_{n}^{d}(s)\right)<1$, we first note that $\left\|u^{s}\right\|_{\infty} \leq$ $C\|\phi\|_{\infty}$ by (2.5). Next, by the boundary Lipschitz estimate, we obtain $\left|\nabla u^{s}(x)\right| \leq$ $C\|\phi\|_{C^{2}\left(\mathbb{T}^{d}\right)}$ if $\operatorname{dist}\left(x, \partial \mathbb{H}_{n}^{d}(s)\right)<1$. This, together with (2.20) and (2.19), proves (2.15).

Finally, we prove the inequality (2.16) by using interpolation and the Sobolev embedding. Precisely, for any $L>0$, it follows from (2.15), (2.9) and interpolation that

$$
\begin{aligned}
\left\|N^{T} \nabla_{\theta} V\right\|_{H^{r+\frac{d}{2}+1}\left(\mathbb{T}^{d} \times[L, L+1]\right)} & \leq C\left\|N^{T} \nabla_{\theta} V\right\|_{L^{2}\left(\mathbb{T}^{d} \times[L, L+1]\right)}^{1-\sigma}\left\|N^{T} \nabla_{\theta} V\right\|_{H^{k-1}\left(\mathbb{T}^{d} \times[L, L+1]\right)}^{\sigma} \\
& \leq C(1+L)^{-(1-\sigma)}\|\phi\|_{C^{k}\left(\mathbb{T}^{d}\right)},
\end{aligned}
$$

where $k=k(d, r, \sigma) \geq 1$ is sufficiently large. It follows from the Sobolev embedding theorem that

$$
\begin{aligned}
\sup _{(\theta, t) \in \mathbb{T}^{d} \times[L, L+1]}\left|N^{T} \nabla_{\theta} \partial_{\theta}^{\alpha} \partial_{t}^{j} V(\theta, t)\right| & \leq C\left\|N^{T} \nabla_{\theta} V\right\|_{H^{r+d / 2+1}\left(\mathbb{T}^{d} \times[L, L+1]\right)} \\
& \leq \frac{C\|\phi\|_{C^{k}\left(\mathbb{T}^{d}\right)}}{(1+L)^{1-\sigma}}
\end{aligned}
$$


which readily implies

$$
\left|N^{T} \nabla_{\theta} \partial_{\theta}^{\alpha} \partial_{t}^{j} V(\theta, t)\right| \leq \frac{C\|\phi\|_{C^{k}\left(\mathbb{T}^{d}\right)}}{(1+t)^{1-\sigma}} \quad \text { for any }(\theta, t) \in \mathbb{T}^{d} \times \mathbb{R}_{+},
$$

where $|\alpha|+j \leq r$. A similar argument gives the pointwise estimate for $\left|\partial_{\theta}^{\alpha} \partial_{t}^{1+j} V\right|$.

Lemma 2.8. Let $V$ be the solution of (2.7) with $n \in \mathbb{S}^{d-1}$. Then

$$
|V|+\left|\nabla_{\theta} V\right| \leq C\|\phi\|_{C^{2}\left(\mathbb{T}^{d}\right)},
$$

where $C$ depends only on $d, m$ and $A$. Moreover, if $n \in \mathbb{S}_{D}^{d-1}$ with Diophantine constant $\varkappa=\varkappa(n)>0$, then for any $|\alpha| \geq 2$ and $0<\sigma<1$,

$$
\left|\partial_{\theta}^{\alpha} V\right| \leq C \varkappa^{-\sigma}\|\phi\|_{C^{k}\left(\mathbb{T}^{d}\right)},
$$

where $k=k(d,|\alpha|, \sigma)>1$ and $C$ depends only on $d, m,|\alpha|, \sigma$ and $A$.

Proof. Again, the desired estimates for $V$ will be reduced to estimates for solutions $u^{s}$ of (2.17), where $V$ and $u^{s}$ are related by (2.18). First, since $\left\|u^{s}\right\|_{\infty} \leq C\|\phi\|_{\infty}$, we obtain $|V| \leq C\|\phi\|_{\infty}$. Next, by comparing $u^{s}$ and $u^{s^{\prime}}$ in the common domain, we may deduce from the boundary Lipschitz estimate and the Miranda-Agman maximal principle (2.5) that

$$
\left|u^{s}(x)-u^{s^{\prime}}(x)\right| \leq C\left|s-s^{\prime}\right|\|\phi\|_{C^{2}\left(\mathbb{T}^{d}\right)},
$$

if $x \cdot n<-\max \left\{s, s^{\prime}\right\}$. Observe that, to prove the boundedness of $\nabla_{\theta} V$, it suffices to prove the boundedness of $n \cdot \nabla_{\theta} V$, as $N^{T} \nabla_{\theta} V$ is bounded due to (2.15). To this end, note that

$$
\begin{aligned}
& |V(\theta+r n, t)-V(\theta, t)|=\left|u^{-\theta \cdot n-r}(\theta+r n-t n)-u^{-\theta \cdot n}(\theta-t n)\right| \\
& \leq\left|u^{-\theta \cdot n-r}(\theta+r n-t n)-u^{-\theta \cdot n-r}(\theta-t n)\right|+\left|u^{-\theta \cdot n-r}(\theta-t n)-u^{-\theta \cdot n}(\theta-t n)\right| \\
& \leq|r|\left\|\nabla u^{-\theta \cdot n-r}\right\|_{\infty}+\left\|u^{-\theta \cdot n-r}-u^{-\theta \cdot n}\right\|_{\infty} \\
& \leq C|r|\|\phi\|_{C^{2}\left(\mathbb{T}^{d}\right)},
\end{aligned}
$$

where we have used (2.24) for the last step. Dividing by $r$ on both sides and taking the limit as $r \rightarrow 0$, we obtain $\left|n \cdot \nabla_{\theta} V\right| \leq C\|\phi\|_{C^{2}\left(\mathbb{T}^{d}\right)}$. This finishes the proof of (2.22).

Finally, to show (2.23), we use (2.22), (2.10) and an interpolation argument. Precisely, let $L>0$ and $t \in[L, L+1]$,

$$
\begin{aligned}
\sup _{(\theta, t) \in \mathbb{T}^{d} \times[L, L+1]}\left|\partial_{\theta}^{\alpha} V(\theta, t)\right| & \leq C\|V\|_{H^{d / 2+|\alpha|+1}\left(\mathbb{T}^{d} \times[L, L+1]\right)} \\
& \leq C\|V\|_{H^{1}\left(\mathbb{T}^{d} \times[L, L+1]\right)}^{1-\sigma}\|V\|_{H^{r}\left(\mathbb{T}^{d} \times[L, L+1]\right)}^{\sigma} \\
& \leq C \varkappa^{-\sigma}\|\phi\|_{C^{k}\left(\mathbb{T}^{d}\right)},
\end{aligned}
$$

where $|\alpha| \geq 2$ and $r=r(d, \alpha, \sigma), k=k(d,|\alpha|, \sigma)$ are sufficiently large. The desired estimate follows. 
Now we are ready to prove Theorem 2.6.

\section{Proof of Theorem 2.6. Step 1: Set-up and reduction.}

Fix $n_{1}, n_{2} \in \mathbb{S}_{D}^{d-1}$. We may assume that $\delta=\left|n_{1}-n_{2}\right|>0$ is sufficiently small. Let $N_{1}$ and $N_{2}$ be the $d \times(d-1)$ matrices such that both $M_{1}=\left(N_{1},-n_{1}\right)$ and $M_{2}=\left(N_{2},-n_{2}\right)$ are orthogonal matrices. Recall that solution $V_{1}$ (resp. $V_{2}$ ) of (2.7), associated with $n_{1}$ (resp. $n_{2}$ ), is independent of the choices of $N_{1}$ (resp. $N_{2}$ ). So without loss of generality, we may assume $\left|N_{1}-N_{2}\right| \leq C \delta$. To be precise, we write down the systems for $V_{1}$ and $V_{2}$ as follows:

$$
\left\{\begin{aligned}
-\left(\begin{array}{c}
N_{1}^{T} \nabla_{\theta} \\
\partial_{t}
\end{array}\right) \cdot B_{1}\left(\begin{array}{c}
N_{1}^{T} \nabla_{\theta} \\
\partial_{t}
\end{array}\right) V_{1}=0 & \text { in } \mathbb{T}^{d} \times(0, \infty), \\
V_{1}=\phi & \text { on } \mathbb{T}^{d} \times\{0\}
\end{aligned}\right.
$$

and

$$
\left\{\begin{aligned}
-\left(\begin{array}{c}
N_{2}^{T} \nabla_{\theta} \\
\partial_{t}
\end{array}\right) \cdot B_{2}\left(\begin{array}{c}
N_{2}^{T} \nabla_{\theta} \\
\partial_{t}
\end{array}\right) V_{2}=0 & \text { in } \mathbb{T}^{d} \times(0, \infty), \\
V_{2}=\phi & \text { on } \mathbb{T}^{d} \times\{0\},
\end{aligned}\right.
$$

where $B_{\ell}(\theta, t)=M_{\ell}^{T} A^{*}\left(\theta-t n_{\ell}\right) M_{\ell}$ for $\ell=1,2$ and $\phi=-\chi_{k}^{* \beta}$. In view of Theorem 2.5, to show (2.14), it suffices to prove that

$$
\int_{\mathbb{T}^{d}}\left|\partial_{t} V_{1}(\theta, 0)-\partial_{t} V_{2}(\theta, 0)\right|^{2} d \theta \leq C \varkappa^{-2 \sigma}\left|n_{1}-n_{2}\right|^{2}
$$

Define $W=V_{1}-V_{2}$. Observe that

$$
\int_{\mathbb{T}^{d}}\left|\partial_{t} W(\theta, 0)\right|^{2} d \theta \leq 2 \int_{0}^{1} \int_{\mathbb{T}^{d}}\left|\partial_{t} W(\theta, t)\right|^{2} d \theta d t+2 \int_{0}^{1} \int_{\mathbb{T}^{d}}\left|\partial_{t}^{2} W(\theta, t)\right|^{2} d \theta d t .
$$

Thus, the estimate (2.27) is further reduced to that for the two integrals in the RHS of (2.28). We may assume that $\varkappa\left(n_{1}\right) \geq \varkappa\left(n_{2}\right)$ and thus $\varkappa=\varkappa\left(n_{1}\right)$.

\section{Step 2: Estimate for $\partial_{t} W$.}

Note that $W$ satisfies $W(\theta, 0)=0$ and

$$
\begin{aligned}
& -\left(\begin{array}{c}
N_{2}^{T} \nabla_{\theta} \\
\partial_{t}
\end{array}\right) \cdot B_{2}\left(\begin{array}{c}
N_{2}^{T} \nabla_{\theta} \\
\partial_{t}
\end{array}\right) W \\
& =-\left(\begin{array}{c}
N_{2}^{T} \nabla_{\theta} \\
\partial_{t}
\end{array}\right) \cdot B_{2}\left(\begin{array}{c}
N_{2}^{T} \nabla_{\theta} \\
\partial_{t}
\end{array}\right) V_{1} \\
& =\left[\left(\begin{array}{c}
N_{1}^{T} \nabla_{\theta} \\
\partial_{t}
\end{array}\right) \cdot B_{1}\left(\begin{array}{c}
N_{1}^{T} \nabla_{\theta} \\
\partial_{t}
\end{array}\right)-\left(\begin{array}{c}
N_{2}^{T} \nabla_{\theta} \\
\partial_{t}
\end{array}\right) \cdot B_{2}\left(\begin{array}{c}
N_{2}^{T} \nabla_{\theta} \\
\partial_{t}
\end{array}\right)\right] V_{1} .
\end{aligned}
$$


By using

$$
\begin{aligned}
& \left(\begin{array}{c}
N_{1}^{T} \nabla_{\theta} \\
\partial_{t}
\end{array}\right) \cdot B_{1}\left(\begin{array}{c}
N_{1}^{T} \nabla_{\theta} \\
\partial_{t}
\end{array}\right)-\left(\begin{array}{c}
N_{2}^{T} \nabla_{\theta} \\
\partial_{t}
\end{array}\right) \cdot B_{2}\left(\begin{array}{c}
N_{2}^{T} \nabla_{\theta} \\
\partial_{t}
\end{array}\right) \\
& =-\left(\begin{array}{c}
N_{2}^{T} \nabla_{\theta} \\
\partial_{t}
\end{array}\right) \cdot B_{2}\left(\begin{array}{c}
\left(N_{2}^{T}-N_{1}^{T}\right) \nabla_{\theta} \\
0
\end{array}\right)-\left(\begin{array}{c}
N_{2}^{T} \nabla_{\theta} \\
\partial_{t}
\end{array}\right) \cdot\left(B_{2}-B_{1}\right)\left(\begin{array}{c}
N_{1}^{T} \nabla_{\theta} \\
\partial_{t}
\end{array}\right) \\
& \quad+\left(\begin{array}{c}
\left(N_{2}^{T}-N_{1}^{T}\right) \nabla_{\theta} \\
0
\end{array}\right) \cdot B_{1}\left(\begin{array}{c}
N_{1}^{T} \nabla_{\theta} \\
\partial_{t}
\end{array}\right),
\end{aligned}
$$

the RHS of (2.29) can be written as

$$
\left(\begin{array}{c}
N_{2}^{T} \nabla_{\theta} \\
\partial_{t}
\end{array}\right) \cdot\left(G_{1}+G_{2}\right)+H
$$

where

$$
\begin{aligned}
G_{1} & =-B_{2}\left(\begin{array}{c}
\left(N_{2}^{T}-N_{1}^{T}\right) \nabla_{\theta} \\
0
\end{array}\right) V_{1}, \\
G_{2} & =-\left(B_{2}-B_{1}\right)\left(\begin{array}{c}
N_{1}^{T} \nabla_{\theta} \\
\partial_{t}
\end{array}\right) V_{1}, \\
H & =\left(\begin{array}{c}
\left(N_{2}^{T}-N_{1}^{T}\right) \nabla_{\theta} \\
0
\end{array}\right) \cdot B_{1}\left(\begin{array}{c}
N_{1}^{T} \nabla_{\theta} \\
\partial_{t}
\end{array}\right) V_{1} .
\end{aligned}
$$

Therefore, the equation (2.29) is reduced to

$$
-\left(\begin{array}{c}
N_{2}^{T} \nabla_{\theta} \\
\partial_{t}
\end{array}\right) \cdot B_{2}\left(\begin{array}{c}
N_{2}^{T} \nabla_{\theta} \\
\partial_{t}
\end{array}\right) W=\left(\begin{array}{c}
N_{2}^{T} \nabla_{\theta} \\
\partial_{t}
\end{array}\right) \cdot G+H
$$

where $G=G_{1}+G_{2}$.

Lemma 2.9. [21, Remark 6.3] Let $n \in \mathbb{S}^{d-1}$ and $U$ be a smooth solution of

$$
-\left(\begin{array}{c}
N^{T} \nabla_{\theta} \\
\partial_{t}
\end{array}\right) \cdot B\left(\begin{array}{c}
N^{T} \nabla_{\theta} \\
\partial_{t}
\end{array}\right) U=\left(\begin{array}{c}
N^{T} \nabla_{\theta} \\
\partial_{t}
\end{array}\right) \cdot G+H
$$

with $U(\cdot, 0)=0$. Assume that

$$
\sup _{t>0, \theta \in \mathbb{T}^{d}}(1+t)\left\{\left|N^{T} \nabla_{\theta} U(\theta, t)\right|+\left|\partial_{t} U(\theta, t)\right|+|G(\theta, t)|+(1+t)|H(\theta, t)|\right\}<\infty
$$

Then, for any $0<\sigma<1$,

$$
\int_{0}^{\infty} \int_{\mathbb{T}^{d}}\left(\left|N^{T} \nabla_{\theta} U\right|^{2}+\left|\partial_{t} U\right|^{2}\right) t^{\sigma-1} d \theta d t \leq C \int_{0}^{\infty} \int_{\mathbb{T}^{d}}\left(|G|^{2}+t^{2}|H|^{2}\right) t^{\sigma-1} d \theta d t
$$


Applying Lemma 2.9 to the system (2.31), we obtain

$$
\begin{aligned}
\int_{0}^{\infty} \int_{\mathbb{T}^{d}} & \left(\left|N_{2}^{T} \nabla_{\theta} W\right|^{2}+\left|\partial_{t} W\right|^{2}\right) t^{\sigma-1} d \theta d t \\
& \leq C \int_{0}^{\infty} \int_{\mathbb{T}^{d}}\left(|G|^{2}+t^{2}|H|^{2}\right) t^{\sigma-1} d \theta d t .
\end{aligned}
$$

Hence, it suffices to estimate the integrals involving $G$ and $H$ in (2.35).

Estimate for the integral with $G_{1}$ : By the estimates for $\left|\nabla V_{1}\right|$ in $(2.22)$ and (2.10), we have

$$
\left|G_{1}(\theta, t)\right| \leq C \delta\left|\nabla_{\theta} V_{1}(\theta, t)\right| \leq C \delta \cdot 1^{1-\sigma}\left[\varkappa^{-1}(1+\varkappa t)^{-\ell}\right]^{\sigma}
$$

for any $0<\sigma<1$. It follows that

$$
\begin{aligned}
\int_{0}^{\infty} \int_{\mathbb{T}^{d}}\left|G_{1}\right|^{2} t^{\sigma-1} d \theta d t & \leq C \delta^{2} \varkappa^{-2 \sigma} \int_{0}^{\infty} \frac{d t}{t^{1-\sigma}(1+\varkappa t)^{2 \ell \sigma}} \\
& \leq C \delta^{2} \varkappa^{-3 \sigma} \int_{0}^{\infty} \frac{d t}{t^{1-\sigma}(1+t)^{2 \ell \sigma}} \\
& \leq C \delta^{2} \varkappa^{-3 \sigma}
\end{aligned}
$$

where we can simply choose $\ell=1$ to ensure the convergence of the integral in the righthand side.

Estimate for the integral with $G_{2}$ : Note that an interpolation between (2.15) and (2.10) implies

$$
\left|N_{1}^{T} \nabla_{\theta} V_{1}(\theta, t)\right|+\left|\partial_{t} V_{1}(\theta, t)\right| \leq C(1+t)^{\sigma-1}(1+\varkappa t)^{-\ell \sigma}
$$

Also note that $\left|B_{1}(\theta, t)-B_{2}(\theta, t)\right| \leq C t \delta$. It follows that

$$
\begin{aligned}
\int_{0}^{\infty} \int_{\mathbb{T}^{d}}\left|G_{2}\right|^{2} t^{\sigma-1} d \theta d t & \leq C \delta^{2} \int_{0}^{\infty} \frac{t^{1+\sigma} d t}{(1+t)^{2(1-\sigma)}(1+\varkappa t)^{2 \ell \sigma}} \\
& \leq C \delta^{2} \varkappa^{-3 \sigma}
\end{aligned}
$$

where we need to choose $\ell=2$.

Estimate for the integral with $H$ : Observe that

$$
\begin{aligned}
\int_{0}^{\infty} \int_{\mathbb{T}^{d}}|H|^{2} t^{1+\sigma} d \theta d t \leq C & \delta^{2} \int_{0}^{\infty} \int_{\mathbb{T}^{d}}\left(\left|N_{1}^{T} \nabla_{\theta} V_{1}\right|^{2}+\left|\partial_{t} V_{1}\right|^{2}\right) t^{1+\sigma} d \theta d t \\
& +C \delta^{2} \int_{0}^{\infty} \int_{\mathbb{T}^{d}}\left(\left|N_{1}^{T} \nabla_{\theta} \nabla_{\theta} V_{1}\right|^{2}+\left|\partial_{t} \nabla_{\theta} V_{1}\right|^{2}\right) t^{1+\sigma} d \theta d t
\end{aligned}
$$

The first term in the RHS is bounded by $\delta^{2} \varkappa^{-3 \sigma}$ by using (2.37). To handle the second integral, we apply the interpolation theorem between (2.16) and (2.10) to obtain

$$
\left|N_{1}^{T} \nabla_{\theta} \nabla_{\theta} V_{1}(\theta, t)\right|+\left|\partial_{t} \nabla_{\theta} V_{1}(\theta, t)\right| \leq C(1+t)^{-(1-\sigma)^{2}}(1+\varkappa t)^{-\ell \sigma}
$$


Thus, the second term is bounded by

$$
C \delta^{2} \int_{0}^{\infty} \frac{t^{1+\sigma} d t}{(1+t)^{2(1-2 \sigma)}(1+\varkappa t)^{2 \ell \sigma}} \leq C \delta^{2} \varkappa^{-5 \sigma}
$$

where we have chosen $\ell=3$.

By combining the estimates above with (2.35), we obtain

$$
\int_{0}^{1} \int_{\mathbb{T}^{d}}\left(\left|N_{2}^{T} \nabla_{\theta} W\right|^{2}+\left|\partial_{t} W\right|^{2}\right) d \theta d t \leq C_{\sigma} \delta^{2} \varkappa^{-5 \sigma}
$$

\section{Step 3: Estimate for $\partial_{t}^{2} W$.}

Let $N_{2 j}$ denote the $j$ th column of $N_{2}$ and define $\nabla_{2 j}=N_{2 j}^{T} \cdot \nabla_{\theta}$ for $1 \leq j \leq d-1$. Note that $\nabla_{2 j}$ is the $j$ th component of $N_{2}^{T} \nabla_{\theta}$. Then we apply $\nabla_{2 j}$ to (2.31) and obtain

$$
\begin{aligned}
-\left(\begin{array}{c}
N_{2}^{T} \nabla_{\theta} \\
\partial_{t}
\end{array}\right) \cdot B_{2}\left(\begin{array}{c}
N_{2}^{T} \nabla_{\theta} \\
\partial_{t}
\end{array}\right) \nabla_{2 j} W=\left(\begin{array}{c}
N_{2}^{T} \nabla_{\theta} \\
\partial_{t}
\end{array}\right) \cdot \nabla_{2 j} G+\nabla_{2 j} H \\
\\
+\left(\begin{array}{c}
N_{2}^{T} \nabla_{\theta} \\
\partial_{t}
\end{array}\right) \cdot \nabla_{2 j} B_{2}\left(\begin{array}{c}
N_{2}^{T} \nabla_{\theta} \\
\partial_{t}
\end{array}\right) W
\end{aligned}
$$

on $\mathbb{T}^{d} \times \mathbb{R}_{+}$and $\nabla_{2 j} W=0$ on $\mathbb{T}^{d} \times\{0\}$. Let $\eta(t)$ be a cut-off function such that $\eta(t)=1$ for $t \in[0,1], \eta(t)=0$ for $t \in[2, \infty), 0 \leq \eta(t) \leq 1$ and $|\nabla \eta| \leq C$. Now integrating (2.41) against $\eta^{2} \nabla_{2 j} W$, we derive from integration by parts that

$$
\begin{aligned}
& \int_{0}^{1} \int_{\mathbb{T}^{d}}\left(\left|N_{2}^{T} \nabla_{\theta} \nabla_{2 j} W\right|^{2}+\left|\partial_{t} \nabla_{2 j} W\right|^{2}\right) d \theta d t \\
& \quad \leq C \int_{0}^{2} \int_{\mathbb{T}^{d}}\left(\left|\nabla_{2 j} G\right|^{2}+\left|\nabla_{2 j} H\right|^{2}+\left|N_{2}^{T} \nabla_{\theta} W\right|^{2}+\left|\partial_{t} W\right|^{2}\right) d \theta d t \\
& \quad \leq C \varkappa^{-5 \sigma} \delta^{2}
\end{aligned}
$$

where we have used the fact $\left|\nabla_{2 j} W\right| \leq\left|N_{2}^{T} \nabla_{\theta} W\right|$. Consequently,

$$
\int_{0}^{1} \int_{\mathbb{T}^{d}}\left(\left|N_{2}^{T} \nabla_{\theta} \otimes N_{2}^{T} \nabla_{\theta} W\right|^{2}+\left|\partial_{t} N_{2}^{T} \nabla_{\theta} W\right|^{2}\right) d \theta d t \leq C \varkappa^{-5 \sigma} \delta^{2} .
$$

Now observe that

$$
\begin{aligned}
& \left(\begin{array}{c}
N_{2}^{T} \nabla_{\theta} \\
\partial_{t}
\end{array}\right) \cdot B_{2}\left(\begin{array}{c}
N_{2}^{T} \nabla_{\theta} \\
\partial_{t}
\end{array}\right) W \\
& =\left(\begin{array}{c}
N_{2}^{T} \nabla_{\theta} \\
\partial_{t}
\end{array}\right) B_{2} \cdot\left(\begin{array}{c}
N_{2}^{T} \nabla_{\theta} \\
\partial_{t}
\end{array}\right) W+B_{2}:\left[\left(\begin{array}{c}
N_{2}^{T} \nabla_{\theta} \\
\partial_{t}
\end{array}\right) \otimes\left(\begin{array}{c}
N_{2}^{T} \nabla_{\theta} \\
\partial_{t}
\end{array}\right)\right] W \\
& =\left(\begin{array}{c}
N_{2}^{T} \nabla_{\theta} \\
\partial_{t}
\end{array}\right) B_{2} \cdot\left(\begin{array}{c}
N_{2}^{T} \nabla_{\theta} \\
\partial_{t}
\end{array}\right) W+B_{2}:\left[\begin{array}{cc}
N_{2}^{T} \nabla_{\theta} \otimes N_{2}^{T} \nabla_{\theta} & N_{2}^{T} \nabla_{\theta} \partial_{t} \\
\left(N_{2}^{T} \nabla_{\theta} \partial_{t}\right)^{T} & 0
\end{array}\right] W+b_{2, d d} \partial_{t}^{2} W,
\end{aligned}
$$


where $b_{2, d d}=\left(b_{2, d d}^{\alpha \beta}\right)_{1 \leq \alpha, \beta \leq m}$ is positive due to the strong ellipticity condition. This gives

$$
\begin{gathered}
b_{2, d d} \partial_{t}^{2} W=-\left(\begin{array}{c}
N_{2}^{T} \nabla_{\theta} \\
\partial_{t}
\end{array}\right) B_{2} \cdot\left(\begin{array}{c}
N_{2}^{T} \nabla_{\theta} \\
\partial_{t}
\end{array}\right) W-B_{2}:\left[\begin{array}{cc}
N_{2}^{T} \nabla_{\theta} \otimes N_{2}^{T} \nabla_{\theta} & N_{2}^{T} \nabla_{\theta} \partial_{t} \\
\left(N_{2}^{T} \nabla_{\theta} \partial_{t}\right)^{T} & 0
\end{array}\right] W \\
-\left(\begin{array}{c}
N_{2}^{T} \nabla_{\theta} \\
\partial_{t}
\end{array}\right) \cdot G-H .
\end{gathered}
$$

Note that $\left|\left(b_{2, d d}\right)^{-1}\right| \leq C$. Thus, it follows from (2.40), (2.42) and the pointwise estimates of $G$ and $H$ for $t \in[0,1]$ that

$$
\int_{0}^{1} \int_{\mathbb{T}^{d}}\left|\partial_{t}^{2} W\right|^{2} d \theta d t \leq C \delta^{2} \varkappa^{-5 \sigma}
$$

This completes the proof of Theorem 2.6.

Proof of Theorem 1.1. Note that $\partial \Omega$ is locally differential homeomorphic to $\mathbb{R}^{d-1}$. Thus, in view of Theorem 2.6, it suffices to prove the following claim: Let $F \in L^{1}\left(\mathbb{R}^{d-1} ; \mathbb{R}^{m}\right)$ and $G \in L^{p}\left(\mathbb{R}^{d-1}\right)$ for some $1<p<\infty$. Suppose that for a.e. $x \in \mathbb{R}^{d-1}$,

$$
|F(x)-F(y)| \leq|x-y||G(x)|, \quad \text { for a.e. } y \in \mathbb{R}^{d-1} .
$$

Then

$$
\left(\int_{\mathbb{R}^{d-1}}|\nabla F|^{p}\right)^{1 / p} \leq C\left(\int_{\mathbb{R}^{d-1}}|G|^{p}\right)^{1 / p}
$$

where $C$ depends only on $d$ and $p$. Indeed, if the claim holds, then it follows from Theorem 2.6 that

$$
\left(\int_{\partial \Omega}\left|\nabla_{\tan } \bar{f}\right|^{p}\right)^{1 / p} \leq C\left(\int_{\mathbb{T}^{d}}\|f(\cdot, y)\|_{C^{1}(\partial \Omega)}^{2} d y\right)^{1 / 2}\left(\int_{\partial \Omega}[\varkappa(n(x))]^{-\sigma p} d x\right)^{1 / p}
$$

for any $0<\sigma<1$. Recall that $[\varkappa(n(x))]^{-1} \in L^{q}(\partial \Omega)$ for any $q<d-1$. Thus, for any $p<\infty$, we choose $\sigma \in(0,1)$ so small that $\sigma p<d-1$. As a result, we obtain

$$
\left(\int_{\partial \Omega}\left|\nabla_{\tan } \bar{f}\right|^{p}\right)^{1 / p} \leq C\left(\int_{\mathbb{T}^{d}}\|f(\cdot, y)\|_{C^{1}(\partial \Omega)}^{2} d y\right)^{1 / 2}
$$

for any $p<\infty$. Note that $\bar{f}$ is bounded. We may conclude that $\bar{f} \in W^{1, p}\left(\partial \Omega ; \mathbb{R}^{m}\right)$ and (1.8) holds.

It remains to prove the claim. Let $\varphi \in C_{0}^{\infty}(B(0,1))$ and $\int_{\mathbb{R}^{d-1}} \varphi=1$. Set $\varphi_{\varepsilon}(x)=$ $\varepsilon^{1-d} \varphi(x / \varepsilon)$. Define for any $\varepsilon>0$,

$$
F_{\varepsilon}(x)=\int_{\mathbb{R}^{d-1}} F(y) \varphi_{\varepsilon}(x-y) d y .
$$


Clearly, $F_{\varepsilon}$ is smooth and $F_{\varepsilon} \rightarrow F$ in $L^{1}\left(\mathbb{R}^{d-1} ; \mathbb{R}^{m}\right)$ as $\varepsilon \rightarrow 0$. Moreover, for any $z \in$ $B(x, \varepsilon)$,

$$
\begin{aligned}
\nabla F_{\varepsilon}(x) & =\int_{\mathbb{R}^{d-1}} F(y) \nabla \varphi_{\varepsilon}(x-y) d y \\
& =\int_{\mathbb{R}^{d-1}}(F(y)-F(z)) \nabla \varphi_{\varepsilon}(x-y) d y .
\end{aligned}
$$

Using the assumption (2.44),

$$
\begin{aligned}
\left|\nabla F_{\varepsilon}(x)\right| & \leq f_{B(x, \varepsilon)}|G(z)| \int_{B(x, \varepsilon)}|y-z|\left|\nabla \varphi_{\varepsilon}(x-y)\right| d y d z \\
& \leq C f_{B(x, \varepsilon)}|G(z)| d z \\
& \leq C\left(f_{B(x, \varepsilon)}|G(z)|^{p} d z\right)^{1 / p} .
\end{aligned}
$$

Thus, by Fubini's Theorem, for any $\varepsilon>0$

$$
\left(\int_{\mathbb{R}^{d-1}}\left|\nabla F_{\varepsilon}(x)\right|^{p} d x\right)^{1 / p} \leq C\left(\int_{\mathbb{R}^{d-1}}|G(z)|^{p} d z\right)^{1 / p} .
$$

Since $\nabla F_{\varepsilon} \rightarrow \nabla F$ in the sense of distribution as $\varepsilon \rightarrow 0$, (2.45) follows from (2.49).

\section{Regularity for Neumann problems}

As in the case of Dirichlet problems, to establish the regularity of $\bar{g}_{i j}$, we use an explicit formula for $\bar{g}_{i j}$ previously discovered in [21]. It involves a family of Neumann problems in the half-spaces:

$$
\left\{\begin{aligned}
\mathcal{L}_{1}^{*} u^{s} & =0 & & \text { in } \mathbb{H}_{n}^{d}(s), \\
n \cdot A^{*} \nabla u^{s} & =T \cdot \nabla \phi & & \text { on } \partial \mathbb{H}_{n}^{d}(s),
\end{aligned}\right.
$$

where $T$ is a constant tangential vector, i.e., $T \cdot n=0$, with $|T| \leq 1$. We assume that $\phi \in C^{\infty}\left(\mathbb{T}^{d} ; \mathbb{R}^{m}\right)$.

As far as we know, for arbitrary $n \in \mathbb{S}^{d-1}$, the solvability of (3.1) is not clear. But for $n \in \mathbb{S}_{D}^{d-1}$, it was shown in [21] that (3.1) is solvable by lifting the problem to a $(d+1)$ dimensional system in the upper half-space, in a manner similar to the case of Dirichlet condition. More precisely, we seek a solution in the form of

$$
u^{s}(x)=U(x-(x \cdot n+s) n,-(x \cdot n+s)),
$$


where $U$ is a solution of the Neumann problem:

$$
\left\{\begin{aligned}
-\left(\begin{array}{c}
N^{T} \nabla_{\theta} \\
\partial_{t}
\end{array}\right) \cdot B\left(\begin{array}{c}
N^{T} \nabla_{\theta} \\
\partial_{t}
\end{array}\right) U=0 & \text { in } \mathbb{T}^{d} \times \mathbb{R}_{+}, \\
-e_{d+1} \cdot B\left(\begin{array}{c}
N^{T} \nabla_{\theta} \\
\partial_{t}
\end{array}\right) U=T \cdot \nabla_{\theta} \phi & \text { on } \mathbb{T}^{d} \times\{0\},
\end{aligned}\right.
$$

with $B(\theta, t)=M^{T} A^{*}(\theta-t n) M$ and $M=(N,-n)$ being an orthogonal matrix. The solvability of (3.3) and related estimates are addressed below.

Lemma 3.1. [21, Proposition 3.6] Suppose that $n$ satisfies the Diophantine condition with constant $\varkappa>0$. Then the Neumann problem (3.3) has a smooth solution $U$, and the solution is unique, up to a constant under the condition that $U \in L^{\infty}\left(\mathbb{T}^{d} \times \mathbb{R}_{+}\right)$, $\nabla_{\theta} U \in L^{2}\left(\mathbb{T}^{d} \times \mathbb{R}_{+}\right)$and $\partial_{t} U \in L^{2}\left(\mathbb{T}^{d} \times \mathbb{R}_{+}\right)$. Moreover, the solution satisfies

$$
\int_{0}^{\infty} \int_{\mathbb{T}^{d}}\left\{\left|N^{T} \nabla_{\theta} \partial_{\theta}^{\alpha} \partial_{t}^{j} U\right|^{2}+\left|\partial_{\theta}^{\alpha} \partial_{t}^{1+j} U\right|^{2}\right\} d \theta d t \leq C\|\phi\|_{C^{|\alpha|+j+1}\left(\mathbb{T}^{d}\right)}^{2}
$$

for any $|\alpha|, j \geq 0$, where $C$ depends only on $d, m,|\alpha|, j$, and $A$. Furthermore, there exists a constant vector $U_{\infty}$ such that for any $|\alpha|, j, \ell \geq 0$,

$$
\left|N^{T} \nabla_{\theta} \partial_{\theta}^{\alpha} \partial_{t}^{j} U\right|+\left|\partial_{\theta}^{\alpha} \partial_{t}^{1+j} U\right|+\varkappa\left|\partial_{\theta}^{\alpha}\left(U-U_{\infty}\right)\right| \leq \frac{C_{\ell}\|\phi\|_{C^{k}\left(\mathbb{T}^{d}\right)}}{(1+\varkappa t)^{\ell}}
$$

where $k=k(|\alpha|, j, \ell, d)$ and $C_{\ell}$ depends only on $d, m,|\alpha|, j, \ell$, and $A$.

Remark 3.2. Lemma 3.1 gives the existence of solutions to (3.1) for $s \in \mathbb{R}$ and $n \in \mathbb{S}_{D}^{d-1}$ via (3.2). Moreover, by the (large-scale) uniform boundary Lispchitz estimates for Neumann conditions [16, 7], the solution satisfying the sublinear growth as $x \cdot n \rightarrow-\infty$ is unique up to a constant.

Recall that $\mathbb{S}_{D}^{d-1}$ has full surface measure of $\mathbb{S}^{d-1}$. An expression for $\bar{g}_{i j}$ defined a.e. on $\mathbb{S}^{d-1}$ is formulated in [21] and summarized below.

Theorem 3.3. Let $g=\left\{g_{i j}\right\}$, where $g_{i j} \in C^{\infty}\left(\partial \Omega \times \mathbb{T}^{d} ; \mathbb{R}^{m}\right)$. Then, for any $x \in \partial \Omega$ with $n=n(x) \in \mathbb{S}_{D}^{d-1}$,

$$
\bar{g}_{j k}^{\gamma}(x)=n_{i} \widehat{a}_{j i}^{\alpha \gamma} h^{\alpha \beta} T_{\ell r} \cdot \int_{\mathbb{T}^{d}}\left[e_{k} \delta^{\nu \beta}+\nabla_{\theta} \chi_{k}^{* \nu \beta}(\theta)+\nabla_{\theta} U_{n, k}^{\nu \beta}(\theta, 0)\right] g_{\ell r}^{\nu}(x, \theta) d \theta,
$$

where $\left(h^{\alpha \beta}\right)$ denotes the inverse of the $m \times m$ matrix $\left(\widehat{a}_{i j}^{* \alpha \beta} n_{i} n_{j}\right)$ and $U_{n, k}^{\beta}$ is the solution of

$$
\left\{\begin{array}{cc}
-\left(\begin{array}{c}
N^{T} \nabla_{\theta} \\
\partial_{t}
\end{array}\right) \cdot B_{n}\left(\begin{array}{c}
N^{T} \nabla_{\theta} \\
\partial_{t}
\end{array}\right) U_{n, k}^{\beta}=0 & \text { in } \mathbb{T}^{d} \times(0, \infty), \\
-e_{d+1} \cdot B_{n}\left(\begin{array}{c}
N^{T} \nabla_{\theta} \\
\partial_{t}
\end{array}\right) U_{n, k}^{\beta}=\frac{1}{2} T_{i j} \cdot \nabla_{\theta} \phi_{i j, k}^{\beta} & \text { on } \mathbb{T}^{d} \times\{0\}
\end{array}\right.
$$


where $T_{i j}=n_{i} e_{j}-n_{j} e_{i}, B_{n}(\theta, t)=M^{T} A^{*}(\theta-t n) M$, and $\phi_{i j, k}^{\beta}=\left(\phi_{i j, k}^{1 \beta}, \phi_{i j, k}^{2 \beta}, \cdots, \phi_{i j, k}^{m \beta}\right)$ are the 1-periodic smooth functions satisfying

$$
\frac{\partial}{\partial \theta_{i}}\left\{\phi_{i j, k}^{\alpha \beta}\right\}=a_{j k}^{* \alpha \beta}+a_{j \ell}^{* \alpha \gamma} \frac{\partial}{\partial \theta_{\ell}} \chi_{k}^{* \gamma \beta}-\widehat{a}_{j k}^{* \alpha \beta} \quad \text { and } \quad \phi_{i j, k}^{\alpha \beta}=-\phi_{j i, k}^{\alpha \beta} .
$$

We point out that the functions $\phi_{i j, k}^{\beta}$, which are completely determined by $A$, are smooth as long as $A$ is. The equations (3.8) for $\phi_{i j, k}^{\beta}$ will not be used in this paper.

Theorem 3.4. Fix $\sigma \in(0,1)$. Let $x, y \in \partial \Omega$ and $|x-y| \leq c_{0}$. Suppose that $n(x)$, $n(y) \in \mathbb{S}_{D}^{d-1}$. Then

$$
|\bar{g}(x)-\bar{g}(y)| \leq C_{\sigma} \varkappa^{-\sigma}|x-y|\left(\int_{\mathbb{T}^{d}}\|g(\cdot, y)\|_{C^{1}(\partial \Omega)}^{2} d y\right)^{1 / 2},
$$

where $\varkappa=\max \{\varkappa(n(x)), \varkappa(n(y))\}$ and $C_{\sigma}$ depends only on $d, m, \sigma, \lambda$, and $\|A\|_{C^{k}\left(\mathbb{T}^{d}\right)}$ for some $k=k(d, \sigma) \geq 1$.

To prove Theorem 3.4, the following two lemmas will be crucial.

Lemma 3.5. Let $n \in \mathbb{S}_{D}^{d-1}$ and $U$ be a solution of (3.3) corresponding to $n$. Then

$$
\left|N^{t} \nabla_{\theta} U\right|+\left|\partial_{t} U\right| \leq \frac{C\|\phi\|_{C^{k}\left(\mathbb{T}^{d}\right)}}{1+t}
$$

where $k>d / 2+1$ and $C$ depends only on $d, m$ and $A$. Moreover, for any $0<\sigma<1$,

$$
\left|N^{t} \nabla_{\theta} \partial_{\theta}^{\alpha} \partial_{t}^{j} U\right|+\left|\partial_{\theta}^{\alpha} \partial_{t}^{1+j} U\right| \leq \frac{C_{\sigma}\|\phi\|_{C^{k}\left(\mathbb{T}^{d}\right)}}{(1+t)^{1-\sigma}}
$$

where $k=k(|\alpha|, j, \sigma, d)$ and $C_{\sigma}$ depends only on $d, m,|\alpha|, j, \sigma$ and $A$.

Proof. Let $u^{s}$ be the solution of (3.1), given by (3.2). Then it follows from [21, Theorem 4.1] that

$$
\left|\nabla u^{s}(x)\right| \leq \frac{C\|\phi\|_{\infty}}{|x \cdot n+s|} \quad \text { for } x \cdot n+s<0 .
$$

Observe that (3.2) is equivalent to $U(\theta, t)=u^{-\theta \cdot n}(\theta-t n)$ for any $(\theta, t) \in \mathbb{T}^{d} \times \mathbb{R}_{+}$. It follows that

$$
\left\{\begin{array}{c}
N^{T} \nabla_{\theta} U(\theta, t)=N^{T} \nabla_{x} u^{-\theta \cdot n}(\theta-t n), \\
\partial_{t} U(\theta, t)=-n \cdot \nabla_{x} u^{-\theta \cdot n}(\theta-t n) .
\end{array}\right.
$$

In view of (3.12) and (3.13) we obtain

$$
\left|N^{t} \nabla_{\theta} U(\theta, t)\right|+\left|\partial_{t} U(\theta, t)\right| \leq \frac{C\|\phi\|_{L^{\infty}}}{t} .
$$

This gives (3.10) for $t \geq 1 / 2$. The case $t \in[0,1 / 2]$ follows from (3.4) and the Sobolev embedding theorem in $\mathbb{T}^{d} \times[0,1]$, which requires $k>d / 2+1$.

Finally, the estimate (3.11) follows from (3.10), (3.4) and an interpolation argument, as in the proof of Lemma 2.7. 
Lemma 3.6. Let $n \in \mathbb{S}_{D}^{d-1}$ with Diophantine constant $\varkappa>0$ and $U$ be a solution of (3.3) corresponding to $n$. Then there exists a constant vector $U_{\infty}$ such that for any $0<\sigma<1$ and $|\alpha| \geq 0$

$$
\left|\partial_{\theta}^{\alpha}\left(U-U_{\infty}\right)\right| \leq C_{\sigma} \varkappa^{-\sigma}\|f\|_{C^{k}\left(\mathbb{T}^{d}\right)} .
$$

where $k=k(\alpha, \sigma, d)$ and $C_{\sigma}$ depends only on $d, m, \alpha, \sigma$, and $A$.

Proof. We first observe that it suffices to show $\left|U-U_{\infty}\right| \leq C_{\sigma} \varkappa^{-\sigma}\|f\|_{C^{k}\left(\mathbb{T}^{d}\right)}$ for any $0<\sigma<1$. Then the case $|\alpha|>0$ follows from this and (3.5) by an interpolation argument.

Note that $\left|U-U_{\infty}\right| \rightarrow 0$ as $t \rightarrow \infty$. It follows from (3.5) and (3.10) that

$$
\left|\partial_{t} U(\theta, t)\right| \leq C \frac{\|f\|_{C^{k}\left(\mathbb{T}^{d}\right)}^{1-\sigma}}{(1+t)^{1-\sigma}} \cdot \frac{\|f\|_{C^{k}\left(\mathbb{T}^{d}\right)}^{\sigma}}{(1+\varkappa t)^{\sigma \ell}} .
$$

Hence,

$$
\begin{aligned}
\sup _{t>0}\left|\left(U-U_{\infty}\right)(\theta, t)\right| & \leq \int_{0}^{\infty}\left|\partial_{t} U(\theta, t)\right| d t \\
& \leq C\|f\|_{C^{k}\left(\mathbb{T}^{d}\right)} \int_{0}^{\infty} \frac{d t}{(1+t)^{1-\sigma}(1+\varkappa t)^{\sigma \ell}} \\
& \leq C \varkappa^{-\sigma}\|f\|_{C^{k}\left(\mathbb{T}^{d}\right)} .
\end{aligned}
$$

This completes the proof.

Proof of Theorem 3.4. Step 1: Set-up and reduction. Let $n_{1}=\left(n_{1,1}, \cdots, n_{1, d}\right)$, $n_{2}=\left(n_{2,1}, \cdots, n_{2, d}\right) \in \mathbb{S}_{D}^{d-1}$ and $\delta=\left|n_{1}-n_{2}\right|>0$. Choose $d \times(d-1)$ matrices $N_{1}, N_{2}$ such that both $M_{1}=\left(N_{1},-n_{1}\right)$ and $M_{2}=\left(N_{2},-n_{2}\right)$ are orthogonal and $\left|N_{1}-N_{2}\right| \leq C \delta$. Let $U_{1}, U_{2}$ be solutions of the systems in the form of (3.7) associated with $n_{1}, n_{2}$, respectively, i.e.,

$$
\left\{\begin{array}{cc}
-\left(\begin{array}{c}
N_{1}^{T} \nabla_{\theta} \\
\partial_{t}
\end{array}\right) \cdot B_{1}\left(\begin{array}{c}
N_{1}^{T} \nabla_{\theta} \\
\partial_{t}
\end{array}\right) U_{1}=0 & \text { in } \mathbb{T}^{d} \times(0, \infty), \\
-e_{d+1} \cdot B_{1}\left(\begin{array}{c}
N_{1}^{T} \nabla_{\theta} \\
\partial_{t}
\end{array}\right) U_{1}=T_{1, i j} \cdot \nabla_{\theta} \phi_{i j} & \text { on } \mathbb{T}^{d} \times\{0\}
\end{array}\right.
$$

and

$$
\left\{\begin{array}{cc}
-\left(\begin{array}{c}
N_{2}^{T} \nabla_{\theta} \\
\partial_{t}
\end{array}\right) \cdot B_{2}\left(\begin{array}{c}
N_{2}^{T} \nabla_{\theta} \\
\partial_{t}
\end{array}\right) U_{2}=0 & \text { in } \mathbb{T}^{d} \times(0, \infty), \\
-e_{d+1} \cdot B_{2}\left(\begin{array}{c}
N_{2}^{T} \nabla_{\theta} \\
\partial_{t}
\end{array}\right) U_{2}=T_{2, i j} \cdot \nabla_{\theta} \phi_{i j} & \text { on } \mathbb{T}^{d} \times\{0\},
\end{array}\right.
$$

where $T_{\ell, i j}=n_{\ell, i} e_{j}-n_{\ell, j} e_{i}$ are vectors orthogonal to $n_{\ell}$ and $B_{\ell}(\theta, t)=M_{\ell}^{T} A^{*}\left(\theta-t n_{\ell}\right) M_{\ell}$ for $\ell=1,2$.

Without loss of generality, we may assume that $\varkappa=\varkappa\left(n_{1}\right) \geq \varkappa\left(n_{2}\right)$. In view of the formula (3.6), we only need to show that

$$
\int_{\mathbb{T}^{d}}\left|T_{1, i j} \cdot \nabla_{\theta} U_{1}(\theta, 0)-T_{2, i j} \cdot \nabla_{\theta} U_{2}(\theta, 0)\right|^{2} d \theta \leq C_{\sigma} \varkappa^{-2 \sigma}\left|n_{1}-n_{2}\right|^{2}
$$


for $1 \leq i, j \leq d$. By the triangle inequality,

$$
\begin{aligned}
\int_{\mathbb{T}^{d}} & \left|T_{1, i j} \cdot \nabla_{\theta} U_{1}(\theta, 0)-T_{2, i j} \cdot \nabla_{\theta} U_{2}(\theta, 0)\right|^{2} d \theta \\
& \leq 2 \int_{\mathbb{T}^{d}}\left|\left(T_{1, i j}-T_{2, i j}\right) \cdot \nabla_{\theta} U_{1}(\theta, 0)\right|^{2} d \theta+2 \int_{\mathbb{T}^{d}}\left|T_{2, i j} \cdot \nabla_{\theta}\left(U_{1}(\theta, 0)-U_{2}(\theta, 0)\right)\right|^{2} d \theta \\
& \leq C \varkappa^{-2 \sigma} \delta^{2}+C \int_{\mathbb{T}^{d}}\left|N_{2}^{T} \nabla_{\theta}\left(U_{1}(\theta, 0)-U_{2}(\theta, 0)\right)\right|^{2} d \theta,
\end{aligned}
$$

where in the last inequality we have used (3.15) and the fact that the columns of $N_{2}$ span the subspace orthogonal to $n_{2}$. Furthermore, we let $W=U_{1}-U_{2}$ and note that

$$
\begin{aligned}
& \int_{\mathbb{T}^{d}}\left|N_{2}^{T} \nabla_{\theta} W(\theta, 0)\right|^{2} d \theta \\
& \quad \leq 2 \int_{0}^{1} \int_{\mathbb{T}^{d}}\left|N_{2}^{T} \nabla_{\theta} W(\theta, t)\right|^{2} d \theta d t+2 \int_{0}^{1} \int_{\mathbb{T}^{d}}\left|N_{2}^{T} \nabla_{\theta} \partial_{t} W(\theta, t)\right|^{2} d \theta d t .
\end{aligned}
$$

As a result, it suffices to estimate the two terms in the RHS of the above inequality.

Step 2: Estimate for $N_{2}^{T} \nabla_{\theta} W$.

The argument here is similar to that for Dirichlet problems, with Lemmas 3.1, 3.5 and 3.6 in our disposal. Note that $W$ satisfies

$$
\left\{\begin{aligned}
-\left(\begin{array}{c}
N_{2}^{T} \nabla_{\theta} \\
\partial_{t}
\end{array}\right) \cdot B_{2}\left(\begin{array}{c}
N_{2}^{T} \nabla_{\theta} \\
\partial_{t}
\end{array}\right) W=\left(\begin{array}{c}
N_{2}^{T} \nabla_{\theta} \\
\partial_{t}
\end{array}\right) \cdot G+H & \text { in } \mathbb{T}^{d} \times \mathbb{R}_{+}, \\
-e_{d+1} \cdot B_{2}\left(\begin{array}{c}
N_{2}^{T} \nabla_{\theta} \\
\partial_{t}
\end{array}\right) W=e_{d+1} \cdot G+\left(T_{1, i j}-T_{2, i j}\right) \cdot \nabla_{\theta} \phi_{i j} & \text { on } \mathbb{T}^{d} \times\{0\},
\end{aligned}\right.
$$

where $G=G_{1}+G_{2}$ and $H$ are exactly the same as in (2.30) for Dirichlet problems.

The following two lemmas were proved in [21].

Lemma 3.7. Let $n \in \mathbb{S}_{D}^{d-1}$ and $U$ be a solution of

$$
\left\{\begin{array}{cc}
-\left(\begin{array}{c}
N^{T} \nabla_{\theta} \\
\partial_{t}
\end{array}\right) \cdot B\left(\begin{array}{c}
N^{T} \nabla_{\theta} \\
\partial_{t}
\end{array}\right) U=\left(\begin{array}{c}
N^{T} \nabla_{\theta} \\
\partial_{t}
\end{array}\right) \cdot G & \text { in } \mathbb{T}^{d} \times \mathbb{R}_{+}, \\
-e_{d+1} \cdot B\left(\begin{array}{c}
N^{T} \nabla_{\theta} \\
\partial_{t}
\end{array}\right) U=e_{d+1} \cdot G & \text { on } \mathbb{T}^{d} \times\{0\} .
\end{array}\right.
$$

Assume that

$$
\sup _{t>0, \theta \in \mathbb{T}^{d}}(1+t)\left\{\left|N^{T} \nabla_{\theta} U(\theta, t)\right|+\left|\partial_{t} U(\theta, t)\right|+|G(\theta, t)|\right\}<\infty .
$$

Then, for any $0<\sigma<1$,

$$
\int_{0}^{\infty} \int_{\mathbb{T}^{d}}\left(\left|N^{T} \nabla_{\theta} U\right|^{2}+\left|\partial_{t} U\right|^{2}\right) t^{\sigma-1} d \theta d t \leq C_{\sigma} \int_{0}^{\infty} \int_{\mathbb{T}^{d}}|G|^{2} t^{\sigma-1} d \theta d t .
$$


Lemma 3.8. Let $n \in \mathbb{S}_{D}^{d-1}$ and $U$ be a solution of

$$
\left\{\begin{aligned}
-\left(\begin{array}{c}
N^{T} \nabla_{\theta} \\
\partial_{t}
\end{array}\right) \cdot B\left(\begin{array}{c}
N^{T} \nabla_{\theta} \\
\partial_{t}
\end{array}\right) U=0 & \text { in } \mathbb{T}^{d} \times \mathbb{R}_{+}, \\
-e_{d+1} \cdot B\left(\begin{array}{c}
N^{T} \nabla_{\theta} \\
\partial_{t}
\end{array}\right) U=h & \text { on } \mathbb{T}^{d} \times\{0\} .
\end{aligned}\right.
$$

Assume that

$$
\sup _{t>0, \theta \in \mathbb{T}^{d}}(1+t)\left\{\left|N^{T} \nabla_{\theta} U(\theta, t)\right|+\left|\partial_{t} U(\theta, t)\right|\right\}<\infty .
$$

Then,

$$
\int_{0}^{2} \int_{\mathbb{T}^{d}}\left(\left|N^{T} \nabla_{\theta} U\right|^{2}+\left|\partial_{t} U\right|^{2}\right) d \theta d t \leq C \int_{\mathbb{T}^{d}}|h|^{2} d \theta .
$$

Now we split $W$ as $W=W_{1}+W_{2}+W_{3}$, where

$$
\begin{gathered}
\left\{\begin{array}{cc}
-\left(\begin{array}{c}
N_{2}^{T} \nabla_{\theta} \\
\partial_{t}
\end{array}\right) \cdot B_{2}\left(\begin{array}{c}
N_{2}^{T} \nabla_{\theta} \\
\partial_{t}
\end{array}\right) W_{1}=0 & \text { in } \mathbb{T}^{d} \times \mathbb{R}_{+}, \\
-e_{d+1} \cdot B_{2}\left(\begin{array}{c}
N_{2}^{T} \nabla_{\theta} \\
\partial_{t}
\end{array}\right) W_{1}=\left(T_{1, i j}-T_{2, i j}\right) \cdot \nabla_{\theta} \phi_{i j} & \text { on } \mathbb{T}^{d} \times\{0\},
\end{array}\right. \\
\left\{\begin{array}{cc}
-\left(\begin{array}{c}
N_{2}^{T} \nabla_{\theta} \\
\partial_{t}
\end{array}\right) \cdot B_{2}\left(\begin{array}{c}
N_{2}^{T} \nabla_{\theta} \\
\partial_{t}
\end{array}\right) W_{2}=\left(\begin{array}{c}
N_{2}^{T} \nabla_{\theta} \\
\partial_{t}
\end{array}\right) \cdot G & \text { in } \mathbb{T}^{d} \times \mathbb{R}_{+}, \\
-e_{d+1} \cdot B_{2}\left(\begin{array}{c}
N_{2}^{T} \nabla_{\theta} \\
\partial_{t}
\end{array}\right) W_{2}=e_{d+1} \cdot G & \text { on } \mathbb{T}^{d} \times\{0\},
\end{array}\right.
\end{gathered}
$$

and

$$
\left\{\begin{array}{rr}
-\left(\begin{array}{c}
N_{2}^{T} \nabla_{\theta} \\
\partial_{t}
\end{array}\right) \cdot B_{2}\left(\begin{array}{c}
N_{2}^{T} \nabla_{\theta} \\
\partial_{t}
\end{array}\right) W_{3}=H & \text { in } \mathbb{T}^{d} \times \mathbb{R}_{+}, \\
-e_{d+1} \cdot B_{2}\left(\begin{array}{c}
N_{2}^{T} \nabla_{\theta} \\
\partial_{t}
\end{array}\right) W_{3}=0 & \text { on } \mathbb{T}^{d} \times\{0\} .
\end{array}\right.
$$

Estimate for $W_{1}$. Since $\phi_{i j}$ is smooth, we can show that (3.28) is solvable and the solution $W_{1}$ satisfies (3.26). Thus, by Lemma 3.8,

$$
\begin{aligned}
\int_{0}^{2} \int_{\mathbb{T}^{d}}\left(\left|N_{2}^{T} \nabla_{\theta} W_{1}\right|^{2}+\left|\partial_{t} W_{1}\right|^{2}\right) d \theta d t & \leq C \int_{\mathbb{T}^{d}}\left|T_{1, i j}-T_{2, i j}\right|^{2}\left|\nabla_{\theta} \phi_{i j}\right|^{2} d \theta d t \\
& \leq C \delta^{2} .
\end{aligned}
$$

Estimate for $W_{2}$. By Lemma 3.7, we have

$$
\begin{aligned}
\int_{0}^{\infty} \int_{\mathbb{T}^{d}}\left(\left|N_{2}^{T} \nabla_{\theta} W_{2}\right|^{2}+\left|\partial_{t} W_{2}\right|^{2}\right) t^{\sigma-1} d \theta d t & \leq C \int_{0}^{\infty} \int_{\mathbb{T}^{d}}|G|^{2} t^{\sigma-1} d \theta d t \\
& \leq C \sum_{k=1,2} \int_{0}^{\infty} \int_{\mathbb{T}^{d}}\left|G_{k}\right|^{2} t^{\sigma-1} d \theta d t
\end{aligned}
$$


Using (3.15) and (3.5), we obtain

$$
\left|\nabla_{\theta} U_{1}\right| \leq C \varkappa^{-\sigma(1-\sigma)}\left[\varkappa^{-1}(1+\varkappa t)^{-\ell}\right]^{\sigma} \leq C \varkappa^{-2 \sigma}(1+\varkappa t)^{-\sigma \ell} .
$$

Hence,

$$
\begin{aligned}
\int_{0}^{\infty} \int_{\mathbb{T}^{d}}\left|G_{1}\right|^{2} t^{\sigma-1} d \theta d t & \leq C \varkappa^{-4 \sigma} \delta^{2} \int_{0}^{\infty}(1+\varkappa t)^{-2 \sigma \ell} t^{\sigma-1} d t \\
& \leq C \varkappa^{-5 \sigma} \delta^{2}
\end{aligned}
$$

Similarly, by (3.10) and (3.5), we have

$$
\left|N_{1}^{T} \nabla_{\theta} U_{1}\right|+\left|\partial_{t} U_{1}\right| \leq C(1+t)^{1-\sigma}(1+\varkappa t)^{-\sigma \ell} .
$$

It follows that

$$
\begin{aligned}
\int_{0}^{\infty} \int_{\mathbb{T}^{d}}\left|G_{2}\right|^{2} t^{\sigma-1} d \theta d t & \leq C \delta^{2} \int_{0}^{\infty} t^{2}(1+t)^{2 \sigma-2}(1+\varkappa t)^{-2 \sigma \ell} t^{\sigma-1} d t \\
& \leq C \varkappa^{-3 \sigma} \delta^{2}
\end{aligned}
$$

As a result, we may conclude that

$$
\int_{0}^{\infty} \int_{\mathbb{T}^{d}}\left(\left|N_{2}^{T} \nabla_{\theta} W_{2}\right|^{2}+\left|\partial_{t} W_{2}\right|^{2}\right) t^{\sigma-1} d \theta d t \leq C \varkappa^{-5 \sigma} \delta^{2} .
$$

Estimate for $W_{3}$. The estimate for $W_{3}$ can be reduced to the first two cases. Let

$$
\widetilde{H}(\theta, t)=-\int_{t}^{\infty} H(\theta, s) d s .
$$

Note that $\widetilde{H}$ is bounded for all $(\theta, t) \in \mathbb{T}^{d} \times \mathbb{R}_{+}$. Write

$$
H(\theta, t)=\partial_{t} \widetilde{H}(\theta, t)=\left(\begin{array}{c}
N_{2}^{T} \nabla_{\theta} \\
\partial_{t}
\end{array}\right) \cdot\left(\begin{array}{c}
0 \\
\widetilde{H}(\theta, t)
\end{array}\right) .
$$

Then, we can further decompose $W_{3}$ into $W_{3}=W_{31}+W_{32}$, where

$$
\left\{\begin{array}{cc}
-\left(\begin{array}{c}
N_{2}^{T} \nabla_{\theta} \\
\partial_{t}
\end{array}\right) \cdot B_{2}\left(\begin{array}{c}
N_{2}^{T} \nabla_{\theta} \\
\partial_{t}
\end{array}\right) W_{31}=\left(\begin{array}{c}
N_{2}^{T} \nabla_{\theta} \\
\partial_{t}
\end{array}\right) \cdot\left(\begin{array}{c}
0 \\
\widetilde{H}(\theta, t)
\end{array}\right) & \text { in } \mathbb{T}^{d} \times \mathbb{R}_{+} \\
-e_{d+1} \cdot B_{2}\left(\begin{array}{c}
N_{2}^{T} \nabla_{\theta} \\
\partial_{t}
\end{array}\right) W_{32}=e_{d+1} \cdot\left(\begin{array}{c}
0 \\
\widetilde{H}(\theta, t)
\end{array}\right) & \text { on } \mathbb{T}^{d} \times\{0\}
\end{array}\right.
$$

and

$$
\begin{cases}-\left(\begin{array}{c}
N_{2}^{T} \nabla_{\theta} \\
\partial_{t}
\end{array}\right) \cdot B_{2}\left(\begin{array}{c}
N_{2}^{T} \nabla_{\theta} \\
\partial_{t}
\end{array}\right) W_{32}=0 & \text { in } \mathbb{T}^{d} \times \mathbb{R}_{+}, \\
-e_{d+1} \cdot B_{2}\left(\begin{array}{c}
N_{2}^{T} \nabla_{\theta} \\
\partial_{t}
\end{array}\right) W_{32}=-e_{d+1} \cdot\left(\begin{array}{c}
0 \\
\widetilde{H}(\theta, t)
\end{array}\right) & \text { on } \mathbb{T}^{d} \times\{0\} .\end{cases}
$$


Now by applying Lemma 3.7 for $W_{31}$, we obtain

$$
\int_{0}^{\infty} \int_{\mathbb{T}^{d}}\left(\left|N_{2}^{T} \nabla_{\theta} W_{31}\right|^{2}+\left|\partial_{t} W_{31}\right|^{2}\right) t^{\sigma-1} d \theta d t \leq C \int_{0}^{\infty} \int_{\mathbb{T}^{d}}|\widetilde{H}|^{2} t^{\sigma-1} d \theta d t .
$$

It follows from Hardy's inequality (see [22, p.272]) that

$$
\begin{aligned}
\int_{0}^{\infty} \int_{\mathbb{T}^{d}}|\widetilde{H}|^{2} t^{\sigma-1} d \theta d t & =\int_{\mathbb{T}^{d}} \int_{0}^{\infty}\left|\int_{t}^{\infty} H(\theta, s) d s\right|^{2} t^{\sigma-1} d t d \theta \\
& \leq \frac{4}{(1-\sigma)^{2}} \int_{\mathbb{T}^{d}} \int_{0}^{\infty}|H(\theta, t)|^{2} t^{\sigma-1+2} d t d \theta
\end{aligned}
$$

Consequently,

$$
\int_{0}^{\infty} \int_{\mathbb{T}^{d}}\left(\left|N_{2}^{T} \nabla_{\theta} W_{31}\right|^{2}+\left|\partial_{t} W_{31}\right|^{2}\right) t^{\sigma-1} d \theta d t \leq C \int_{0}^{\infty} \int_{\mathbb{T}^{d}}|H|^{2} t^{1+\sigma} d \theta d t .
$$

For $W_{32}$, using Lemma 3.8 and Hölder's inequality, we have

$$
\begin{aligned}
\int_{0}^{2} \int_{\mathbb{T}^{d}} & \left(\left|N_{2}^{T} \nabla_{\theta} W_{32}\right|^{2}+\left|\partial_{t} W_{32}\right|^{2}\right) d \theta d t \\
& \leq C \int_{\mathbb{T}^{d}}|\widetilde{H}(\theta, 0)|^{2} d \theta \\
& \leq C \int_{\mathbb{T}^{d}}\left|\int_{0}^{\infty}\right| H(\theta, t)|d t|^{2} d \theta \\
& \leq C \int_{\mathbb{T}^{d}}^{\infty} \int_{0}^{\infty}|H(\theta, t)|^{2}(1+t)^{2-\alpha} d t \int_{0}^{\infty}(1+t)^{\alpha-2} d t d \theta \\
& \leq C \int_{0}^{\infty} \int_{\mathbb{T}^{d}}(1+t)^{2}|H(\theta, t)|^{2} t^{-\alpha} d \theta d t .
\end{aligned}
$$

Therefore,

$$
\begin{aligned}
\int_{0}^{2} \int_{\mathbb{T}^{d}} & \left(\left|N_{2}^{T} \nabla_{\theta} W_{3}\right|^{2}+\left|\partial_{t} W_{3}\right|^{2}\right) d \theta d t \\
& \leq C \int_{0}^{\infty} \int_{\mathbb{T}^{d}}(1+t)^{2}|H|^{2} t^{\sigma-1} d \theta d t \\
& \leq C \delta^{2} \int_{0}^{\infty}(1+t)^{2-2(\sigma-1)^{2}}(1+\varkappa t)^{-2 \sigma \ell} t^{\sigma-1} d t \\
& \leq C \varkappa^{-5 \sigma} \delta^{2}
\end{aligned}
$$

where in the last inequality we have chosen $\ell \geq 2$.

Summing up the estimates for $W_{k}$, we arrive at

$$
\int_{0}^{2} \int_{\mathbb{T}^{d}}\left(\left|N_{2}^{T} \nabla_{\theta} W\right|^{2}+\left|\partial_{t} W\right|^{2}\right) d \theta d t \leq C \varkappa^{-5 \sigma} \delta^{2}
$$


which proves the first part of (3.20), as $\sigma \in(0,1)$ can be arbitrarily small.

Step 3: Estimate for $N_{2}^{T} \nabla_{\theta} \partial_{t} W$.

The argument is similar to Step 3 in the proof of Theorem 2.6. Let $N_{2 k}$ denote the $k$ th column of $N_{2}$, and define the $k$ th component of $N_{2}^{T} \nabla_{\theta}$ by $\nabla_{2 k}=N_{2 k}^{T} \cdot \nabla_{\theta}$. We apply $\nabla_{2 k}$ to (3.21) and obtain

$$
\left\{\begin{array}{c}
-\left(\begin{array}{c}
N_{2}^{T} \nabla_{\theta} \\
\partial_{t}
\end{array}\right) \cdot B_{2}\left(\begin{array}{c}
N_{2}^{T} \nabla_{\theta} \\
\partial_{t}
\end{array}\right) \nabla_{2 k} W=\left(\begin{array}{c}
N_{2}^{T} \nabla_{\theta} \\
\partial_{t}
\end{array}\right) \cdot \nabla_{2 k} G+\nabla_{2 k} H \\
+\left(\begin{array}{c}
N_{2}^{T} \nabla_{\theta} \\
\partial_{t}
\end{array}\right) \cdot \nabla_{2 k} B_{2}\left(\begin{array}{c}
N_{2}^{T} \nabla_{\theta} \\
\partial_{t}
\end{array}\right) W \\
\text { in } \mathbb{T}^{d} \times \mathbb{R}_{+}, \\
-e_{d+1} \cdot B_{2}\left(\begin{array}{c}
N_{2}^{T} \nabla_{\theta} \\
\partial_{t}
\end{array}\right) \nabla_{2 k} W=e_{d+1} \cdot \nabla_{2 k} G+\nabla_{2 k} h \\
+e_{d+1} \cdot \nabla_{2 k} B_{2}\left(\begin{array}{c}
N_{2}^{T} \nabla_{\theta} \\
\partial_{t}
\end{array}\right) W \\
\text { on } \mathbb{T}^{d} \times\{0\},
\end{array}\right.
$$

where $h=\left(T_{1, i j}-T_{2, i j}\right) \cdot \nabla_{\theta} f_{i j}$. Let $\eta(t)$ be a cut-off function such that $\eta(t)=1$ for $t \in[0,1], \eta(t)=0$ for $t \in[2, \infty), 0 \leq \eta(t) \leq 1$ and $|\nabla \eta| \leq C$. Now by integrating (3.41) against $\nabla_{2 k}\left(W \eta^{2}\right)$, we derive from integration by parts that

$$
\begin{aligned}
& \int_{0}^{1} \int_{\mathbb{T}^{d}}\left(\left|N_{2}^{T} \nabla_{\theta} \nabla_{2 k} W\right|^{2}+\left|\partial_{t} \nabla_{2 k} W\right|^{2}\right) d \theta d t \\
& \quad \leq C \int_{0}^{2} \int_{\mathbb{T}^{d}}\left(\left|\nabla_{2 k} G\right|^{2}+\left|\nabla_{2 k} H\right|^{2}+\left|N_{2}^{T} \nabla_{\theta} W\right|^{2}+\left|\partial_{t} W\right|^{2}\right) d \theta d t+C\|h\|_{H^{1}\left(\mathbb{T}^{d}\right)}^{2} \\
& \quad \leq C \varkappa^{-5 \sigma} \delta^{2} .
\end{aligned}
$$

Consequently,

$$
\int_{0}^{1} \int_{\mathbb{T}^{d}}\left(\left|N_{2}^{T} \nabla_{\theta} \otimes N_{2}^{T} \nabla_{\theta} W\right|^{2}+\left|\partial_{t} N_{2}^{T} \nabla_{\theta} W\right|^{2}\right) d \theta d t \leq C \varkappa^{-5 \sigma} \delta^{2},
$$

which finishes the proof.

Proof of Theorem 1.2. With Theorem 3.4 at our disposal, the proof of Theorem 1.2 is identical to that of Theorem 1.1.

\section{References}

[1] H. Aleksanyan, Regularity of boundary data in periodic homogenization of elliptic systems in layered media, Manuscripta Math. (2016), 1-32. 
[2] _ Slow convergence in periodic homogenization problems for divergence-type elliptic operators, SIAM J. Math. Anal. 48 (2016), no. 5, 3345-3382. MR 3549023

[3] H. Aleksanyan, H. Shahgholian, and P. Sjölin, Applications of Fourier analysis in homogenization of Dirichlet problem I. Pointwise estimates, J. Differential Equations 254 (2013), no. 6, 2626-2637. MR 3016217

[4] _ Applications of Fourier analysis in homogenization of Dirichlet problem III: Polygonal domains, J. Fourier Anal. Appl. 20 (2014), no. 3, 524-546. MR 3217486

[5] _ Applications of Fourier analysis in homogenization of the Dirichlet problem: $L^{p}$ estimates, Arch. Ration. Mech. Anal. 215 (2015), no. 1, 65-87. MR 3296144

[6] G. Allaire and M. Amar, Boundary layer tails in periodic homogenization, ESAIM Control Optim. Calc. Var. 4 (1999), 209-243 (electronic). MR 1696289

[7] S. N. Armstrong and Z. Shen, Lipschitz estimates in almost-periodic homogenization, Comm. Pure Appl. Math. 69 (2016), no. 10, 1882-1923. MR 3541853

[8] S.N. Armstrong, T. Kuusi, J.-C. Mourrat, and C. Prange, Quantitative analysis of boundary layers in periodic homogenization, arXiv:1607.06716v2 (2016).

[9] M. Avellaneda and F. Lin, Compactness methods in the theory of homogenization, Comm. Pure Appl. Math. 40 (1987), no. 6, 803-847. MR 910954 (88i:35019)

[10] Sunhi Choi and Inwon C. Kim, Homogenization for nonlinear PDEs in general domains with oscillatory Neumann boundary data, J. Math. Pures Appl. (9) 102 (2014), no. 2, 419-448. MR 3227328

[11] William M. Feldman, Homogenization of the oscillating Dirichlet boundary condition in general domains, J. Math. Pures Appl. (9) 101 (2014), no. 5, 599-622. MR 3192425

[12] William M. Feldman and Inwon C. Kim, Continuity and discontinuity of the boundary layer tail, Ann. Sci. Éc. Norm. Supér. (4) (To appear).

[13] William M. Feldman, Inwon C. Kim, and Panagiotis E. Souganidis, Quantitative homogenization of elliptic partial differential equations with random oscillatory boundary data, J. Math. Pures Appl. (9) 103 (2015), no. 4, 958-1002. MR 3318176

[14] D. Gérard-Varet and N. Masmoudi, Homogenization in polygonal domains, J. Eur. Math. Soc. (JEMS) 13 (2011), no. 5, 1477-1503. MR 2825170

[15] _ Homogenization and boundary layers, Acta Math. 209 (2012), no. 1, 133178. MR 2979511

[16] C.. Kenig, F. Lin, and Z. Shen, Homogenization of elliptic systems with Neumann boundary conditions, J. Amer. Math. Soc. 26 (2013), no. 4, 901-937. MR 3073881 
[17] C. Kenig, F. Lin, and Z. Shen, Periodic homogenization of Green and Neumann functions, Comm. Pure Appl. Math. 67 (2014), no. 8, 1219-1262. MR 3225629

[18] S. Moskow and M. Vogelius, First-order corrections to the homogenised eigenvalues of a periodic composite medium. A convergence proof, Proc. Roy. Soc. Edinburgh Sect. A 127 (1997), no. 6, 1263-1299. MR 1489436

[19] _ First order corrections to the homogenized eigenvalues of a periodic composite medium. The case of Neumann boundary conditions, Preprint (1997).

[20] C. Prange, Asymptotic analysis of boundary layer correctors in periodic homogenization, SIAM J. Math. Anal. 45 (2013), no. 1, 345-387. MR 3032981

[21] Z. Shen and J. Zhuge, Boundary layers in periodic homogenization of Neumann problems, Comm. Pure Appl. Math. (To appear).

[22] E. M. Stein, Singular integrals and differentiability properties of functions, Princeton Mathematical Series, No. 30, Princeton University Press, Princeton, N.J., 1970. MR 0290095

[23] J. Zhuge, Homogenization and boundary layers in domains of finite type, arXiv:1612.05383v2 (2017).

Zhongwei Shen, Department of Mathematics, University of Kentucky, Lexington, Kentucky 40506, USA.

E-mail: zshen2@uky.edu

Jinping Zhuge, Department of Mathematics, University of Kentucky, Lexington, Kentucky 40506, USA.

E-mail: jinping.zhuge@uky.edu

July 30, 2021 